\title{
Orbital-free approximations to the kinetic-energy density in exchange-correlation MGGA functionals: tests on solids
}

\author{
Fabien Tran, Péter Kovács, Leila Kalantari, Georg K. H. Madsen, and Peter Blaha \\ Institute of Materials Chemistry, Vienna University of Technology, \\ Getreidemarkt 9/165-TC, A-1060 Vienna, Austria
}

\begin{abstract}
A recent study of Mejia-Rodriguez and Trickey [Phys. Rev. A 96, 052512 (2017)] showed that the deorbitalization procedure (replacing the exact Kohn-Sham kinetic-energy density by an approximate orbital-free expression) applied to exchange-correlation functionals of the meta-generalized gradient approximation (MGGA) can lead to important changes in the results for molecular properties. For the present work, the deorbitalization of MGGA functionals is further investigated by considering various properties of solids. It is shown that depending on the MGGA, common orbitalfree approximations to the kinetic-energy density can be sufficiently accurate for the lattice constant, bulk modulus, and cohesive energy. For the band gap, calculated with the modified Becke-Johnson MGGA potential, the deorbitalization has a larger impact on the results.
\end{abstract}

\section{INTRODUCTION}

Kohn-Sham density functional theory ${ }^{1,2}$ (KS-DFT) is a computationally efficient quantum method, which allows the treatment of molecules, surfaces, and solids containing up to several thousands of atoms. KS-DFT is particularly fast when the exchange and correlation (xc) effects are treated at the semilocal level of approximation. The drawback is, however, that there can be some degree of uncertainty in the results with semilocal methods 3,4 The most simple semilocal functional $E_{\mathrm{xc}}$ is the local density approximation (LDA),$\stackrel{2,5,6}{ }$ which is purely a functional of the electron density $\rho=\sum_{i=1}^{N}\left|\psi_{i}\right|^{2}$. Higher accuracy can be obtained by using functionals of the generalized gradient approximation (GGA) $)^{-10}$ which depend additionally on the first derivative of $\rho(\nabla \rho)$. Nowadays, the most advanced and accurate semilocal functionals are the so-called meta-GGA (MGGA) $\stackrel{11}{11}$ which, in addition of $\rho$ and $\nabla \rho$, depend also on the positive-definite KS kinetic-energy density (KED)

$$
t^{\mathrm{KS}}(\mathbf{r})=\frac{1}{2} \sum_{i=1}^{N} \nabla \psi_{i}^{*}(\mathbf{r}) \cdot \nabla \psi_{i}(\mathbf{r})
$$

and/or the second derivative of $\rho\left(\nabla^{2} \rho\right)$ :

$$
E_{\mathrm{xc}}^{\mathrm{MGGA}}=\int \varepsilon_{\mathrm{xc}}\left(\rho(\mathbf{r}), \nabla \rho(\mathbf{r}), \nabla^{2} \rho(\mathbf{r}), t^{\mathrm{KS}}(\mathbf{r})\right) d^{3} r .
$$

Considering how constructing a functional using more ingredients brings more flexibility to it, MGGA functionals should be universally more accurate than LDA and GGA functionals. As with GGA functionals, a plethora of MGGA functionals have been proposed (see Ref. 11 for an exhaustive list) and among the recent ones, SCAN ${ }^{12}$ and $\mathrm{TM}^{13}$ for instance, have shown to be accurate for many types of systems and properties. ${ }^{14}-18$

As discussed in detail in Ref. 11, most MGGA functionals depend only on the $\mathrm{KED} t^{\mathrm{KS}}$, while only very few use also (or only) $\nabla^{2} \rho$. One of the main reasons for not using $\nabla^{2} \rho$ in $E_{\mathrm{xc}}$ are the difficulties encountered when calculating the potential (i.e., the functional derivative of $\left.E_{\mathrm{xc}}\right)$ for self-consistent calculations. Indeed, the presence of $\nabla^{2} \rho$ in $E_{\mathrm{xc}}$ means that the potential contains a term, $\nabla^{2}\left(\partial \varepsilon_{\mathrm{xc}} / \partial\left(\nabla^{2} \rho\right)\right)$, that involves the third and fourth derivatives of $\rho$ (see Ref. 19) which may lead to numerical problems like a greater sensitivity to the integration grid. ${ }^{19-22}$ (To our knowledge, only Ref. 23 reports an implementation of $\nabla^{2} \rho$-MGGA with integration by part of the relevant Hamiltonian matrix elements $^{24}$ to avoid the third and fourth derivatives of $\rho$.) As a comparison, a GGA potential involves only the first and second derivatives of $\rho$ (or only the first if integration by part in the Hamiltonain matrix ${ }^{25}$ is done), and a $t^{\mathrm{KS}}$-dependency in a MGGA functional leads to an additional (non-multiplicative) term in the potential, $-(1 / 2) \nabla \cdot\left(\left(\partial \varepsilon_{\mathrm{xc}} / \partial t^{\mathrm{KS}}\right) \nabla \psi_{i}\right)$, that involves the derivatives of $\psi_{i}$ up to the second order (or only the first if integration by part in the Hamiltonian matrix ${ }^{24}$ is done). Therefore, MGGA calculations have been done using mostly $t^{\mathrm{KS}}$-MGGAs and are becoming increasingly popular (see Refs. $26-29$ for recent works reporting selfconsistent implementations for periodic solids). Furthermore, from the theoretical point of view a benefit of using $t^{\mathrm{KS}}$ is that regions of space dominated by a single orbital can be detected (see, e.g., Ref. 30).

On the other hand, $\nabla^{2} \rho$-MGGAs have the advantage to be explicit functionals of $\rho$ such that the functional derivative leads to a true KS (i.e., multiplicative) potential, which is not the case with $t^{\mathrm{KS}}$-MGGAs. Also, except for the problems with the high derivatives of $\rho$ mentioned above, a new self-consistent implementation of MGGAs should be easier for $\nabla^{2} \rho$-MGGAs. Thus, from the fundamental and practical point of views, $\nabla^{2} \rho$-MGGAs are still of interest and worth to be further considered as done in recent works. $21,22,31$

In particular, Mejia-Rodriguez and Trickey ${ }^{22}$ investigated the effect of replacing the exact orbital-dependent $t^{\mathrm{KS}}$ in existing $t^{\mathrm{KS}}$-MGGA functionals by some orbitalfree $(\mathrm{OF})$ approximations $t^{\mathrm{OF}}$. They called this procedure deorbitalization, meaning that a $t^{\mathrm{KS}}-\mathrm{MGGA}$ is transformed into an explicit density functional $\nabla^{2} \rho$ MGGA. The properties that they considered are the heat 
of formation, bond lengths, and vibration frequencies of molecules. This study showed that the replacement $t^{\mathrm{KS}} \rightarrow t^{\mathrm{OF}}$ can have some impact on the results depending on the xc-MGGA or the OF KED. For instance, the average error for the heat of formation is in some cases only slightly modified, while in some other cases it is increased by one order of magnitude. Also, it seems that none of the OF KED they considered, including the two new ones proposed by Mejia-Rodriguez and Trickey, leads to reasonably small changes in all cases. For the present study, we pursue the investigations on the deorbitalization procedure by considering properties of solids. Several $t^{\mathrm{KS}}$-MGGA energy functionals will be deorbitalized and tested on the lattice constant, bulk modulus, and cohesive energy, while the deorbitalization of the modified Becke-Johnson potentia ${ }^{32}$ will be considered for the electronic structure.

The structure of the paper is the following. Section $\amalg$ provides a brief description of the theory and the computational details. In Sec. III the results obtained with the deorbitalized MGGAs are presented and discussed, while Sec. IV provides some analysis and Sec. V gives the summary of this work.

\section{THEORY AND COMPUTATIONAL DETAILS}

\section{A. Orbital-free kinetic energy densities}

In the KS-DFT method,, 2 the noninteracting kinetic energy component of the total energy is given by $T_{\mathrm{s}}^{\mathrm{KS}}=$ $\int t^{\mathrm{KS}} d^{3} r$, where $t^{\mathrm{KS}}$ is given by Eq. (11). Note that another common expression for the integrand in $T_{\mathrm{s}}^{\mathrm{KS}}$ is $t^{\mathrm{KS}^{\prime}}=-(1 / 2) \sum_{i=1}^{N} \psi_{i}^{*} \nabla^{2} \psi_{i}$ which is related to $t^{\mathrm{KS}}$ by $t^{\mathrm{KS}}=t^{\mathrm{KS}}-(1 / 4) \nabla^{2} \rho$ and leads to the same value of $T_{\mathrm{S}}^{\mathrm{KS}}$ since the integral of $\nabla^{2} \rho$ is zero. For the development of fully OF DFT methods $33-35$ or in the framework of embedding schemes, $\underline{36} \underline{-39}$ expressions for $T_{\mathrm{s}}$ which are explicit functionals of $\rho$ have been proposed, and as for xcfunctionals, the majority of them are of semilocal type. The most simple is the LDA of Thomas and Fermi 40,41 (TF) which is the exact expression for the homogeneous electron gas and reads

$$
T_{s}^{\mathrm{TF}}=C_{\mathrm{TF}} \int \rho^{5 / 3}(\mathbf{r}) d^{3} r
$$

where $C_{\mathrm{TF}}=(3 / 10)\left(3 \pi^{2}\right)^{2 / 3}$. With respect to the exact values $\left(T_{\mathrm{s}}^{\mathrm{KS}}\right)$, the $\mathrm{TF}$ functional leads to underestimations for atoms ${ }^{42}$ and molecules ${ }^{43}-\underline{46}$ of about $10 \%$. Since the kinetic energy is a major component of the total energy $E_{\text {tot }}$ (from the virial theorem $T_{\mathrm{s}} \approx-E_{\text {tot }}$ ) such errors are extremely large. Much better values for $T_{\mathrm{s}}$ can be obtained with gradient-corrected type (GGA) functionals (errors below $0.5 \%$ for the best ones ${ }^{43}-48$ ):

$$
T_{s}^{\mathrm{GGA}}=C_{\mathrm{TF}} \int \rho^{5 / 3}(\mathbf{r}) F_{s}(s) d^{3} r
$$

where $s=|\nabla \rho| /\left(2\left(3 \pi^{2}\right)^{1 / 3} \rho^{4 / 3}\right)$ is the reduced density gradient and $F_{s}$ is the kinetic enhancement factor for which many forms have been proposed in the literature (see Refs. 46, 48 50 for compilations) like, for instance, those that were obtained using the conjointness conjecture between the exchange and kinetic energy functionals $\underline{47,51,52}$ While GGAs can lead to rather accurate (albeit far from enough for an useful OF DFT method) values of $T_{\mathrm{s}}^{\mathrm{GGA}}$, the GGA KEDs defined as the integrand of Eq. (4) show absolutely no resemblance to Eq. (11) $: 53-56$ This can be understood by considering the density-gradient expansion approximation (GEA) of Eq. (1) which, at the second order, is given by 57,58 (L in GEA2L indicates the presence of $\nabla^{2} \rho$ )

$$
t^{\mathrm{GEA} 2 \mathrm{~L}}(\mathbf{r})=t^{\mathrm{TF}}(\mathbf{r})+\frac{1}{9} t^{\mathrm{W}}(\mathbf{r})+\frac{1}{6} \nabla^{2} \rho(\mathbf{r}),
$$

where $t^{\mathrm{TF}}=C_{\mathrm{TF}} \rho^{5 / 3}$ [the integrand of Eq. (3)] and $t^{\mathrm{W}}=$ $|\nabla \rho|^{2} /(8 \rho)$ is the von Weizsäcker 59 KED. It is only by considering $\nabla^{2} \rho$ in an OF KED $t^{\mathrm{OF}}$ that the shape of $t^{\mathrm{OF}}$ can be made reasonably close to $t^{\mathrm{KS}}$ (see Refs. 53, 54, 60, and 61) and despite some attempts,,$\frac{55}{5}$ it is most likely hopeless to construct a GGA KED that looks similar to $t^{\mathrm{KS}}$.

Thus, one has to consider $\nabla^{2} \rho$-dependent OF KED $t^{\mathrm{OF}}$ for a replacement of $t^{\mathrm{KS}}$ in a $t^{\mathrm{KS}}$-MGGA xc-functional with the hope of not changing much the results. As mentioned above, a term $c \nabla^{2} \rho$ (c is a constant) in the KED [like in Eq. (5)] integrates to zero, but would also not contribute to the kinetic potential $\delta T_{\mathrm{s}} / \delta \rho$ in a OF or embedding scheme since the contribution is $\nabla^{2}\left(\partial\left(c \nabla^{2} \rho\right) / \partial\left(\nabla^{2} \rho\right)\right)=\nabla^{2} c=0$. However, MGGA xc-functionals depend on the KED in a more complicated way such that $c \nabla^{2} \rho$ can not be discarded.

The $\nabla^{2} \rho$-dependent KED $t^{\mathrm{OF}}$ that we will consider for a replacement of $t^{\mathrm{KS}}$ in xc-MGGAs are now listed (more detail can be found in the respective references). GEA2L ${ }^{57.58}$ as given by Eq. (5). TW02L, which consists of the GGA TW02 proposed in Ref. 47 (a reparametrization of the PBE GGA exchange $\frac{9}{}$ with $\kappa=0.8438$ and $\mu=0.2319)$ augmented with $(1 / 6) \nabla^{2} \rho$. PC from Perdew and Constantin, $\underline{\underline{6}}$ which was constructed to recover the fourth-order GEA in the slowly varying density limit and $t^{\mathrm{W}}$ in the rapidly varying limit, as well as to satisfy $t^{\mathrm{W}} \leq t^{\mathrm{PC}}$. CR from Cancio and Redd 62 [Eqs. (20) and (21) in Ref. 62 with $\alpha=4$ ], which was constructed in a rather similar way as PC. GEAloc from Cancio and $\operatorname{Redd}^{62}$ [Eq. (37) in Ref. 62], which has the same form as Eq. (5) but with different (optimized) parameters in front of $t^{\mathrm{W}}$ and $\nabla^{2} \rho$. PCopt and CRopt from MejiaRodriguez and Trickey ${ }^{22}$ that are reoptimized versions of PC and CR, respectively. Many other expressions for $t^{\mathrm{OF}}$ could also be considered, e.g., any of the integrand (augmented by $c \nabla^{2} \rho$ ) of the numerous proposed $T_{s}^{\mathrm{GGA}}$ or those proposed recently in Refs. 63 and 64. Nevertheless, our selection of seven different OF KED should be good enough to give us a general idea of the change in the performance of a xc-MGGA when it is deorbitalized. 
It is important to mention that for all considered $\mathrm{OF}$ $\mathrm{KED}$, we chose to enforce the lower bound $t^{\mathrm{W}} \leq t \underline{65,66}$ Thus, it is in fact

$$
t^{\mathrm{OF}^{\prime}}(\mathbf{r})=\max \left(t^{\mathrm{OF}}(\mathbf{r}), t^{\mathrm{W}}(\mathbf{r})\right)
$$

that replaces $t^{\mathrm{KS}}$ in the MGGA xc-functionals, which is also a way to locally reduce the error in $t^{\mathrm{OF}}$. Note that depending on the MGGA xc-functional, Eq. (6) may be anyway necessary to apply if negative values of $t^{\mathrm{OF}}-t^{\mathrm{W}}$ or $t^{\mathrm{OF}}$ lead to problems like, for instance, under a square root.

We also mention that the generalization of the $\mathrm{OF}$ KED formulas for spin-polarized systems is trivially given by ${ }^{67} t\left[\rho_{\uparrow}, \rho_{\downarrow}\right]=t_{\uparrow}\left[\rho_{\uparrow}\right]+t_{\downarrow}\left[\rho_{\downarrow}\right]$, where $t_{\sigma}\left[\rho_{\sigma}\right]=(1 / 2) t\left[2 \rho_{\sigma}\right]$ with $t\left[2 \rho_{\sigma}\right]$ being the non-spin-polarized formula in which $\rho$ is replaced by $2 \rho_{\sigma}$.

\section{B. MGGA exchange-correlation functionals}

The MGGA xc-energy functionals that we will consider to test the accuracy of OF KED are MVS68 and SCAN, 12 that were used by Mejia-Rodriguez and Trickey ${ }^{22}$ for their molecular tests, as well as TM that was proposed by Tao and Mo $\stackrel{13}{\underline{n}}$ The recent SCAN and TM functionals have been shown to be accurate for many types of systems and properties (see, e.g., Refs. 14 18). Additionally, the modified Becke-Johnson MGGA potential ${ }^{32}$ (mBJLDA, combined with LDA correlation ${ }^{\underline{6}}$ ) will also be used to test the accuracy of OF KED by considering the band gap. The mBJLDA potential, which is based on the BJ potential, $\stackrel{69,70}{ }$ was shown to be much more reliable than the standard LDA and GGA methods for band gap calculations and to lead to values that are in very good agreement with experiment in most cases $\underline{32,71-75}$

With an energy functional (MVS, SCAN, or TM), the closeness between OF KEDs and the exact KS KED is quantified by considering properties that depend on the total energy (lattice constant, bulk modulus, and cohesive energy). With the mBJLDA potential, properties like band structure or electron density are more interesting to look at.

\section{Computational details}

The calculations were done with WIEN2k, $\underline{76}$ which is an all-electron code based on the linearized augmented plane-wave method $\stackrel{77,78}{ }$ Very good parameters were chosen such that the results are well converged. As in our previous work,$\frac{14}{\underline{w}}$ the lattice constant, bulk modulus, and cohesive energy obtained with MGGAs were calculated using the GGA $\mathrm{PBE}^{\underline{9}}$ orbitals and density since in WIEN2k there is no implementation of the (nonmultiplicative) potential corresponding to a MGGA energy functional. As discussed in Ref. 14, the effect of self-consistency on the results should be very small

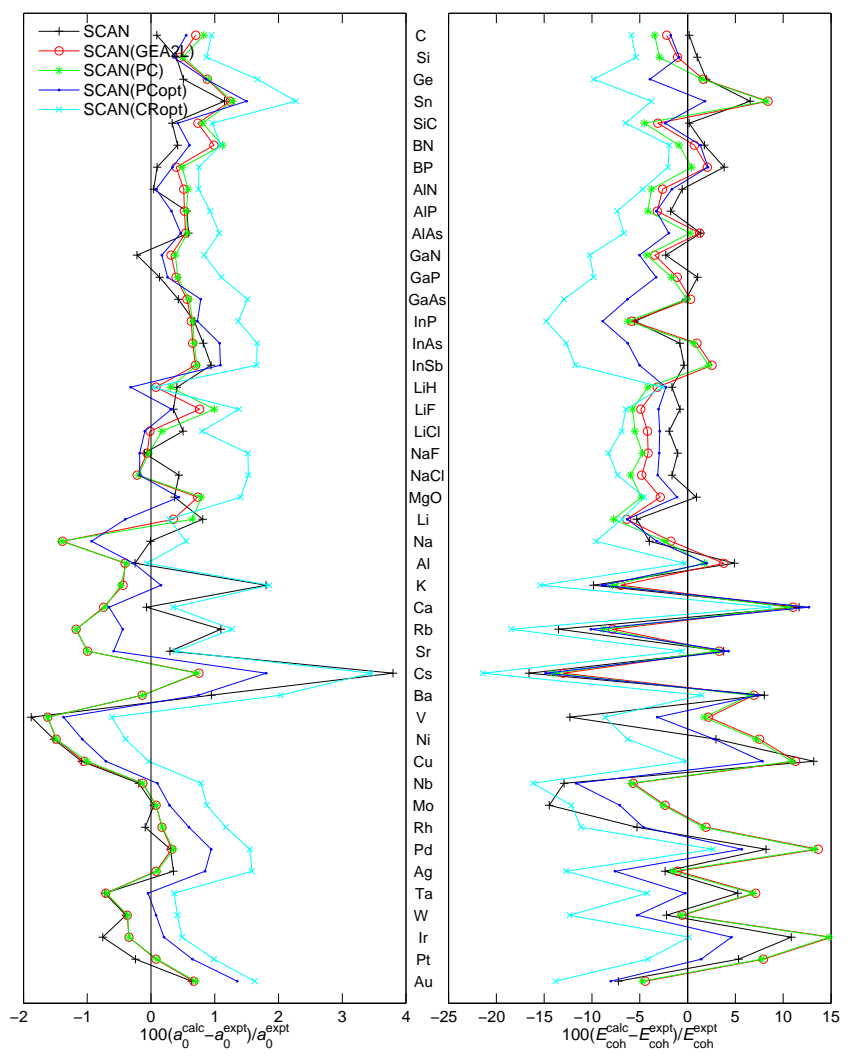

FIG. 1. Relative error (in \%) with respect to experiment ${ }^{79,80}$ in the calculated lattice constant (left panel) and cohesive energy (right panel) for the 44 strongly bound solids.

for strongly bound (i.e., covalent, ionic, metallic) solids. However, self-consistency is expected to affect more the results for weakly bound van der Waals solids. Therefore, this is only via the energy functional that the replacement $t^{\mathrm{KS}} \rightarrow t^{\mathrm{OF}}$ will produce changes in the lattice constant, bulk modulus, and cohesive energy. The calculations of the band gap with the multiplicative mBJLDA potential were done self-consistently.

\section{RESULTS}

\section{A. Lattice constant, bulk modulus, and binding energy}

We start with the results for the equilibrium lattice constant $a_{0}$, bulk modulus $B_{0}$, and cohesive energy $E_{\text {coh }}$ of 44 strongly bound solids (listed in Table S1 of the sup-

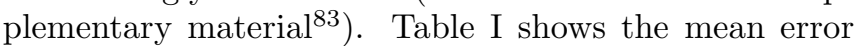
$(\mathrm{ME})$, mean absolute error (MAE), mean relative error (MRE), and mean absolute relative error (MARE) with respect to experiment. The values of $a_{0}, B_{0}$, and $E_{\text {coh }}$ can be found in Tables S1-S9 and Figs. S1-S24 of the supplementary material. 83 The errors obtained with the parent $t^{\mathrm{KS}}$-MGGA, namely, MVS, SCAN, or TM, are considered as the reference that should be reproduced at best by an 
TABLE I. The ME, MAE, MRE, and MARE of the parent $t^{\mathrm{KS}}$-MGGA functionals (MVS, SCAN, and TM) with respect to experiment ${ }^{79,80}$ on the testing set of 44 strongly bound solids for the lattice constant $a_{0}$, bulk modulus $B_{0}$, and cohesive energy $E_{\text {coh }}$. The values for the $t^{\mathrm{OF}}-$ MGGA functionals are also with respect to experiment, but with the value of the parent functional subtracted, e.g., $\mathrm{ME}\left(t^{\mathrm{OF}}-\mathrm{MGGA}\right)-\mathrm{ME}\left(t^{\mathrm{KS}}-\mathrm{MGGA}\right)$. The units of the ME and MAE are $\AA$, GPa, and eV/atom for $a_{0}, B_{0}$, and $E_{\mathrm{coh}}$, respectively, and $\%$ for the MRE and MARE. The large differences with respect to the parent $t^{\mathrm{KS}}-\mathrm{MGGA}$ are underlined. All results were obtained non-self-consistently using PBE orbitals/density.

\begin{tabular}{|c|c|c|c|c|c|c|c|c|c|c|c|c|}
\hline \multirow[b]{2}{*}{ Functional } & \multicolumn{4}{|c|}{$a_{0}$} & \multicolumn{4}{|c|}{$B_{0}$} & \multicolumn{4}{|c|}{$E_{\mathrm{coh}}$} \\
\hline & $\mathrm{ME}$ & MAE & MRE & MARE & $\mathrm{ME}$ & MAE & MRE & MARE & $\mathrm{ME}$ & MAE & MRE & MARE \\
\hline MVS & -0.008 & 0.043 & -0.3 & 0.9 & 12.2 & 13.3 & 8.2 & 12.7 & 0.21 & 0.37 & 5.8 & 9.3 \\
\hline MVS(GEA2L) & $\underline{-0.016}$ & -0.007 & $\underline{-0.3}$ & -0.1 & -4.0 & -3.4 & -1.1 & -3.3 & -0.03 & -0.13 & -1.2 & -3.0 \\
\hline MVS(TW02L) & -0.007 & -0.009 & -0.1 & -0.2 & -4.7 & -3.6 & -2.5 & -4.0 & -0.13 & -0.13 & -3.9 & -2.6 \\
\hline $\operatorname{MVS}(\mathrm{PC})$ & -0.014 & -0.008 & -0.2 & -0.2 & -4.6 & -3.2 & -1.5 & -3.4 & -0.08 & -0.13 & -2.3 & -3.0 \\
\hline MVS(CR) & $\underline{-0.016}$ & -0.007 & $\underline{-0.3}$ & -0.1 & -3.9 & -3.4 & -1.1 & -3.3 & -0.02 & -0.12 & -0.8 & -2.9 \\
\hline MVS(GEAloc) & 0.006 & -0.007 & 0.2 & -0.1 & $\underline{-9.3}$ & $\underline{-5.9}$ & -4.6 & $\underline{-5.2}$ & $\underline{-0.29}$ & -0.15 & $\underline{-6.9}$ & -3.4 \\
\hline MVS(PCopt) & -0.011 & 0.001 & -0.2 & 0.0 & $\underline{-8.4}$ & -3.8 & -3.0 & -3.2 & $\underline{-0.25}$ & -0.08 & $\underline{-5.3}$ & -2.6 \\
\hline MVS(CRopt) & $\underline{0.045}$ & 0.007 & $\underline{1.0}$ & 0.1 & -17.1 & -3.2 & -11.8 & -3.7 & $\underline{-0.59}$ & 0.07 & -14.1 & 1.4 \\
\hline SCAN & 0.018 & 0.030 & 0.3 & 0.6 & 3.5 & 7.4 & -0.5 & 6.5 & -0.02 & 0.19 & -0.7 & 4.9 \\
\hline $\operatorname{SCAN}($ GEA2L $)$ & -0.012 & -0.002 & -0.2 & 0.0 & -4.5 & 2.4 & -0.7 & 1.3 & 0.05 & -0.01 & 1.0 & -0.3 \\
\hline SCAN(TW02L) & -0.007 & -0.001 & -0.1 & 0.0 & $\underline{-5.2}$ & 2.5 & -1.6 & 1.5 & -0.00 & 0.00 & -0.5 & 0.1 \\
\hline $\operatorname{SCAN}(\mathrm{PC})$ & -0.010 & -0.001 & -0.2 & 0.0 & $\underline{-5.0}$ & 2.7 & -1.0 & 1.4 & 0.02 & 0.00 & 0.3 & 0.0 \\
\hline $\mathrm{SCAN}(\mathrm{CR})$ & -0.012 & -0.003 & -0.2 & 0.0 & -4.5 & 2.3 & -0.7 & 1.3 & 0.06 & -0.01 & 1.1 & -0.3 \\
\hline SCAN(GEAloc) & $\underline{0.016}$ & 0.010 & $\underline{0.4}$ & 0.2 & $\underline{-10.4}$ & 3.4 & -3.8 & 2.4 & $\underline{-0.20}$ & 0.06 & -4.5 & 1.3 \\
\hline SCAN(PCopt) & -0.004 & -0.002 & 0.0 & 0.0 & $\underline{-6.4}$ & 0.3 & -1.8 & 0.2 & -0.07 & -0.02 & -1.6 & -0.1 \\
\hline SCAN(CRopt) & $\underline{0.034}$ & $\underline{0.023}$ & $\underline{0.8}$ & $\underline{0.5}$ & $\underline{-11.7}$ & 3.8 & $\underline{-6.2}$ & 3.4 & -0.28 & 0.12 & $\underline{-6.6}$ & 3.0 \\
\hline TM & -0.006 & 0.023 & -0.2 & 0.5 & 2.4 & 6.6 & 2.1 & 6.2 & 0.24 & 0.27 & 6.4 & 7.0 \\
\hline TM(GEA2L) & -0.005 & 0.002 & -0.1 & 0.0 & -0.9 & 0.9 & -0.5 & 0.4 & -0.01 & 0.01 & -0.3 & 0.2 \\
\hline TM(TW02L) & -0.003 & 0.001 & -0.1 & 0.0 & -0.9 & 0.9 & -0.8 & 0.3 & -0.02 & 0.01 & -0.7 & 0.0 \\
\hline $\mathrm{TM}(\mathrm{PC})$ & -0.006 & 0.003 & -0.1 & 0.1 & -0.7 & 1.0 & -0.1 & 0.6 & -0.02 & 0.02 & -0.5 & 0.4 \\
\hline $\mathrm{TM}(\mathrm{CR})$ & -0.005 & 0.002 & -0.1 & 0.0 & -0.8 & 0.9 & -0.5 & 0.4 & -0.00 & 0.01 & -0.1 & 0.2 \\
\hline TM(GEAloc) & -0.010 & 0.003 & -0.2 & 0.1 & -0.4 & 1.6 & 0.9 & 1.2 & -0.01 & 0.03 & 0.2 & 1.1 \\
\hline TM(PCopt) & 0.004 & 0.004 & 0.1 & 0.1 & -2.9 & 1.7 & -1.5 & 0.9 & -0.09 & -0.03 & -2.0 & -0.6 \\
\hline TM(CRopt) & 0.009 & 0.004 & 0.2 & 0.1 & -3.6 & 1.0 & -2.4 & 0.5 & $\underline{-0.13}$ & -0.05 & -2.8 & -1.0 \\
\hline
\end{tabular}

OF $t^{\mathrm{OF}}$-MGGA [denoted $\mathrm{MGGA}(\mathrm{X})$, where $\mathrm{X}$ is one of the OF approximations $t^{\mathrm{OF}}$ mentioned in Sec.IIA. Since the amount of results shown in Table 1 is rather substantial and would make a detailed discussion rather lengthy and tedious, a concise discussion, only in terms of MAE and $\mathrm{ME}$, of the most interesting observations is provided.

In the case of the SCAN and TM xc-functionals, the deorbitalization procedure leads to changes in the MAE and $\mathrm{ME}$ that are the smallest if $t^{\mathrm{KS}}$ is replaced by $t^{\mathrm{GEA} 2 \mathrm{~L}}$, $t^{\mathrm{TW} 02 \mathrm{~L}}, t^{\mathrm{PC}}$, or $t^{\mathrm{CR}}$. The change in the MAE is in most cases below $0.003 \AA$ for $a_{0}, 2.5 \mathrm{GPa}$ for $B_{0}$, and $0.03 \mathrm{eV} /$ atom for $E_{\text {coh }}$, such that it is reasonable to consider the overall (in)accuracy of the xc-functional as unaffected by its deorbitalization. $t^{\mathrm{PCopt}}$ also belongs to the group of the accurate OF KED in the case of SCAN, but not TM especially for the bulk modulus and cohesive energy. If the deorbitalization of SCAN or TM is done with $t^{\text {GEAloc }}$ or $t^{\text {CRopt }}$, then larger changes in the MAE and ME can sometimes, but not systematically, be observed. This seems to be particularly the case with $t^{\mathrm{CRopt}}$, which, for instance, leads for SCAN to changes of $0.023 \AA$ and $3.8 \mathrm{GPa}$ in the MAE of $a_{0}$ and $B_{0}$ respectively. $t^{\mathrm{CRopt}}$ also leads to the largest change in the MAE of $a_{0}$ and $E_{\text {coh }}$ for TM. Thus, replacing $t^{\mathrm{KS}}$ by $t^{\text {GEAloc }}$ or $t^{\mathrm{CRopt}}$, in particular, affects more the accuracy of a functional and would probably change the position of the xc-functional in some performance ranking (see Ref. 14).

Compared to SCAN and TM, the deorbitalization procedure of MVS leads to changes in the MAE that are in general clearly larger. This is due to the analytical form of MVS that depends more strongly on the KED. For instance, for $B_{0}$ there is a decrease in the MAE that is in the range 3.2-5.9 GPa, while for $E_{\text {coh }}$ the MAE of the $t^{\mathrm{OF}}-\mathrm{MVS}$ can be decreased by $0.15 \mathrm{eV} /$ atom [with MVS(GEAloc)] or increased by $0.07 \mathrm{eV} /$ atom [with MVS(CRopt)]. Concerning the ME of MVS, $t^{\mathrm{GEA} 2 \mathrm{~L}}$, $t^{\mathrm{TW} 02 \mathrm{~L}}, t^{\mathrm{PC}}$, and $t^{\mathrm{CR}}$ are more efficient than $t^{\mathrm{GEAloc}}$, $t^{\mathrm{PCopt}}$, and $t^{\mathrm{CRopt}}$ for reproducing the values of MVS. Note that in terms of MAE, MVS(CRopt) seems to be the closest to MVS, but this is fortuitous since the ME 
TABLE II. Equilibrium lattice constant $a_{0}$ (in $\AA$ ) and cohesive energy $E_{\text {coh }}$ (in $\mathrm{meV} /$ atom) of rare-gas solids. The values for the $t^{\mathrm{OF}}$-MGGA functionals are the difference from those obtained with the parent $t^{\mathrm{KS}}$-MGGA, e.g., $a_{0}\left(t^{\mathrm{OF}}\right.$-MGGA $)-$ $a_{0}\left(t^{\mathrm{KS}}-\mathrm{MGGA}\right)$. The reference $\operatorname{CCSD}(\mathrm{T})$ results, which agree closely with experiment, $\stackrel{81}{2}$ are also shown. The large differences with respect to the parent $t^{\mathrm{KS}}$-MGGA are underlined. All results were obtained non-self-consistently using the PBE orbitals/density.

\begin{tabular}{|c|c|c|c|c|c|c|}
\hline \multirow[b]{2}{*}{ Method } & \multicolumn{2}{|c|}{$\mathrm{Ne}$} & \multicolumn{2}{|c|}{ Ar } & \multicolumn{2}{|c|}{$\mathrm{Kr}$} \\
\hline & $a_{0}$ & $E_{\mathrm{coh}}$ & $a_{0}$ & $E_{\mathrm{coh}}$ & $a_{0}$ & $E_{\mathrm{coh}}$ \\
\hline MVS & 4.02 & 59 & 5.41 & 56 & 5.79 & 69 \\
\hline MVS(GEA2L) & $\underline{-0.14}$ & $\underline{41}$ & $\underline{-0.34}$ & $\underline{70}$ & $\underline{-0.30}$ & $\underline{80}$ \\
\hline MVS(TW02L) & -0.03 & 0 & $\underline{-0.21}$ & $\underline{29}$ & $\underline{-0.17}$ & $\underline{33}$ \\
\hline $\operatorname{MVS}(\mathrm{PC})$ & $\underline{-0.15}$ & $\underline{47}$ & $\underline{-0.34}$ & $\underline{75}$ & $\underline{-0.31}$ & $\underline{85}$ \\
\hline $\operatorname{MVS}(\mathrm{CR})$ & $\underline{-0.14}$ & $\underline{41}$ & $\underline{-0.34}$ & $\underline{70}$ & $\underline{-0.30}$ & $\underline{80}$ \\
\hline MVS(GEAloc) & -0.11 & $\underline{31}$ & -0.26 & $\underline{54}$ & -0.19 & $\underline{55}$ \\
\hline MVS(PCopt) & -0.13 & $\underline{38}$ & -0.31 & $\underline{66}$ & -0.23 & $\underline{63}$ \\
\hline MVS(CRopt) & $\underline{0.85}$ & $\underline{-53}$ & $\underline{1.03}$ & $\underline{-48}$ & $\underline{0.75}$ & $\underline{-53}$ \\
\hline SCAN & 4.03 & 54 & 5.31 & 61 & 5.74 & 72 \\
\hline SCAN(GEA2L) & -0.02 & $\underline{11}$ & -0.15 & $\underline{32}$ & $\underline{-0.20}$ & $\underline{50}$ \\
\hline SCAN(TW02L) & 0.03 & $\underline{-6}$ & -0.08 & 9 & -0.15 & 23 \\
\hline $\operatorname{SCAN}(\mathrm{PC})$ & -0.03 & $\underline{15}$ & -0.15 & $\underline{36}$ & $\underline{-0.20}$ & $\underline{54}$ \\
\hline $\operatorname{SCAN}(\mathrm{CR})$ & -0.02 & $\underline{12}$ & -0.15 & $\underline{32}$ & $\underline{-0.20}$ & $\underline{50}$ \\
\hline SCAN(GEAloc) & 0.02 & 5 & -0.06 & 19 & -0.11 & $\underline{33}$ \\
\hline SCAN(PCopt) & -0.03 & $\underline{12}$ & -0.11 & $\underline{28}$ & -0.14 & $\underline{40}$ \\
\hline SCAN(CRopt) & $\underline{0.63}$ & $\underline{-48}$ & $\underline{0.25}$ & $\underline{-37}$ & $\underline{0.26}$ & -37 \\
\hline $\mathrm{TM}$ & 4.05 & 47 & 5.23 & 62 & 5.60 & 82 \\
\hline TM(GEA2L) & -0.00 & $\underline{7}$ & -0.08 & $\underline{22}$ & -0.08 & $\underline{27}$ \\
\hline TM(TW02L) & 0.03 & -5 & -0.05 & 9 & -0.05 & 13 \\
\hline $\mathrm{TM}(\mathrm{PC})$ & -0.03 & $\underline{-8}$ & -0.14 & 12 & -0.14 & 22 \\
\hline $\mathrm{TM}(\mathrm{CR})$ & -0.00 & $\underline{7}$ & -0.08 & $\underline{22}$ & -0.08 & $\underline{27}$ \\
\hline TM(GEAloc) & -0.10 & $\underline{32}$ & $\underline{-0.17}$ & $\underline{56}$ & $\underline{-0.16}$ & $\underline{67}$ \\
\hline TM(PCopt) & -0.01 & -10 & -0.11 & 7 & -0.11 & 15 \\
\hline TM(CRopt) & 0.05 & -4 & -0.01 & 7 & -0.01 & 9 \\
\hline Reference & 4.30 & 26 & 5.25 & 88 & 5.60 & 122 \\
\hline
\end{tabular}

are completely different and of opposite sign.

Figure 1 shows for each solid the relative error in the lattice constant and cohesive energy obtained with the parent SCAN and four of its deorbitalized versions. We can see that the results with $\operatorname{SCAN}($ GEA2L) and SCAN(PC), which are basically the same, are very or fairly close to SCAN results in most cases. The most visible exceptions are the alkali and alkaline earth metals for which the SCAN(CRopt) values follow very closely those obtained with SCAN in particular for $a_{0}$. We also note some large differences in $E_{\text {coh }}$ between SCAN(GEA2L/PC) and SCAN for some of the $3 d$ and $4 d$ transition metals and the ionic compounds. Except for the aforementioned alkali and alkaline earth metals, the lattice constants and cohesive energies obtained with SCAN(CRopt) differ noticeably from SCAN.
SCAN(PCopt) leads to results that are intermediate between SCAN(GEA2L/PC) and SCAN(CRopt).

Thus, in summary the performance of a xc-MGGA functional for strongly bound solids is modified the least when $t^{\mathrm{KS}}$ is replaced by $t^{\mathrm{GEA} 2 \mathrm{~L}}, t^{\mathrm{TW} 02 \mathrm{~L}}, t^{\mathrm{PC}}, t^{\mathrm{CR}}$, or $t^{\mathrm{PCopt}}$. For SCAN and TM the performance is overall little affected by the deorbitalization using one these $\mathrm{OF}$ KED, but more for MVS.

Although the goal of replacing $t^{\mathrm{KS}}$ by $t^{\mathrm{OF}}$ in a xcMGGA was not to improve the agreement with experiment, we mention that it is sometimes the case. By looking at the $\mathrm{MA}(\mathrm{R}) \mathrm{E}$ in Table $\mathrm{I}$, we can see that, for instance, the deorbitalizion of MVS reduces the values for $a_{0}, B_{0}$, and $E_{\text {coh }}$.

In their work, Mejia-Rodriguez and Trickey ${ }^{22}$ reported changes (due to the deorbitalization) in the MAE for bond lengths of molecules that are below $0.002 \AA$ with MVS, which is small. The change in the ME can be larger in some cases, since while the $\mathrm{ME}$ is $-0.0016 \AA$ with MVS, it increases to $0.0069 \AA$ with $\operatorname{MVS}(\mathrm{PC})$, but is rather similar, $-0.0025 \AA$, with MVS(PCopt). The deorbitalisation of SCAN leads to larger changes in the MAE of bond lengths (up to $\sim 0.01 \AA$ ), but not for the $\mathrm{ME}$ since the largest change is $\sim 0.016 \AA$, which is barely larger than for MVS. From these results on molecular bond lengths, $t^{\text {PCopt }}$ seems to be a more accurate $\mathrm{OF}$ KED than the others. This is in line with our observation that $t^{\text {PCopt }}$ is among the most accurate OF KED for the lattice constants of solids. Concerning the heat of formation, 22 the changes in the MAE and ME seem to be in many cases the smallest with $t^{\mathrm{PCopt}}$, as well. For instance, the deorbitalization of SCAN leads to a change in the MAE of +15 and $+0.5 \mathrm{kcal} / \mathrm{mol}$ with $t^{\mathrm{PC}}$ and $t^{\mathrm{PCopt}}$, respectively, and +21 and $+6 \mathrm{kcal} / \mathrm{mol}$ for the $\mathrm{ME}$. We also mention that from the results of Mejia-Rodriguez and Trickey, we can not observe a change in the results due to the deorbitalization that is larger in the case of MVS as we did.

Turning now to weakly bound van der Waals systems, Tables [II and [II show the results for rare-gas (Ne, Ar, and $\mathrm{Kr}$ ) and layered hexagonal solids (graphite, h-BN, $\mathrm{TiS}_{2}, \mathrm{MoTe}_{2}$, and $\mathrm{WSe}{ }_{2}$ ), respectively.

The range of errors in the lattice constant obtained in typical performance tests of DFT functionals on van der Waals systems (see, e.g., Refs. 14, 84 87) is by far much larger than for covalent or ionic solids. Hence, for our systems it should be fair to consider that the performance of a $t^{\mathrm{KS}}$-MGGA (with respect to other functionals) is not really modified by its deorbitalization if the change in the lattice constant is, let us say, below something like (this may be a matter of personal taste) $\sim 0.1-0.15 \AA$ for the rare-gas $\left(a_{0}\right)$ and layered solids $\left(c_{0}\right)$. With this criterion, the results show that the replacement $t^{\mathrm{KS}} \rightarrow t^{\mathrm{OF}}$ in SCAN and TM leads to acceptable changes in the lattice constant in most cases except maybe Kr. With MVS, however, the changes are two or three times larger and unacceptable since they may affect the performance of MVS with respect to other functionals. 
TABLE III. Equilibrium lattice constant $c_{0}$ (in $\AA$ ) and interlayer binding energy $E_{\mathrm{b}}$ (in meV/atom) of layered solids. The values for the $t^{\mathrm{OF}}-\mathrm{MGGA}$ functionals are the difference from those obtained with the parent $t^{\mathrm{KS}}-\mathrm{MGGA}$, e.g., $c_{0}\left(t^{\mathrm{OF}}-\mathrm{MGGA}\right)-$ $c_{0}\left(t^{\mathrm{KS}}-\mathrm{MGGA}\right)$. The intralayer constant $a$ was not optimized, but kept fixed at the experimental value ${ }^{82}$ Reference results $^{82}$ from experiment for $c_{0}$ and from RPA for $E_{\mathrm{b}}$ are also shown. The large differences with respect to the parent $t^{\mathrm{KS}}$-MGGA are underlined. All results were obtained non-self-consistently using PBE orbitals/density.

\begin{tabular}{|c|c|c|c|c|c|c|c|c|c|c|}
\hline \multirow[b]{2}{*}{ Method } & \multicolumn{2}{|c|}{ Graphite } & \multicolumn{2}{|c|}{$\mathrm{h}-\mathrm{BN}$} & \multicolumn{2}{|c|}{$\mathrm{TiS}_{2}$} & \multicolumn{2}{|c|}{$\mathrm{MoTe}_{2}$} & \multicolumn{2}{|c|}{$\mathrm{WSe}_{2}$} \\
\hline & $c_{0}$ & $E_{\mathrm{b}}$ & $c_{0}$ & $E_{\mathrm{b}}$ & $c_{0}$ & $E_{\mathrm{b}}$ & $c_{0}$ & $E_{\mathrm{b}}$ & $c_{0}$ & $E_{\mathrm{b}}$ \\
\hline MVS & 6.60 & 32 & 6.43 & 38 & 5.79 & 30 & 14.66 & 34 & 13.48 & 19 \\
\hline MVS(GEA2L) & $\underline{-0.24}$ & $\underline{13}$ & $\underline{-0.21}$ & 10 & $\underline{-0.19}$ & $\underline{18}$ & $\underline{-0.25}$ & 6 & $\underline{-0.22}$ & $\underline{12}$ \\
\hline MVS(TW02L) & $\underline{-0.22}$ & $\underline{11}$ & $\underline{-0.19}$ & 8 & $\underline{-0.12}$ & 9 & -0.13 & 0 & -0.09 & 5 \\
\hline $\operatorname{MVS}(\mathrm{PC})$ & $\underline{-0.24}$ & $\underline{13}$ & $\underline{-0.20}$ & 10 & $\underline{-0.14}$ & $\underline{17}$ & $\underline{-0.25}$ & 7 & $\underline{-0.22}$ & $\underline{12}$ \\
\hline MVS(CR) & $\underline{-0.24}$ & $\underline{13}$ & $\underline{-0.21}$ & 10 & $\underline{-0.19}$ & $\underline{18}$ & $\underline{-0.25}$ & 6 & $\underline{-0.22}$ & $\underline{12}$ \\
\hline MVS(GEAloc) & $\underline{-0.14}$ & 10 & $\underline{-0.13}$ & 7 & 0.02 & 7 & 0.12 & -2 & 0.07 & 4 \\
\hline MVS(PCopt) & $\underline{-0.13}$ & 10 & $\underline{-0.12}$ & 7 & -0.07 & $\underline{11}$ & 0.01 & 2 & 0.02 & 7 \\
\hline MVS(CRopt) & 0.02 & -1 & $\underline{0.14}$ & -7 & $\underline{0.28}$ & -11 & $\underline{0.37}$ & -13 & $\underline{0.59}$ & -8 \\
\hline SCAN & 6.94 & 20 & 6.79 & 21 & 5.93 & 21 & 14.75 & 30 & 13.68 & 17 \\
\hline $\operatorname{SCAN}(\mathrm{GEA} 2 \mathrm{~L})$ & $\underline{-0.13}$ & 4 & -0.10 & 5 & $\underline{-0.12}$ & $\underline{12}$ & $\underline{-0.33}$ & 8 & $\underline{-0.26}$ & 10 \\
\hline SCAN(TW02L) & -0.10 & 2 & -0.08 & 3 & -0.09 & 8 & $\underline{-0.31}$ & 5 & $\underline{-0.23}$ & 7 \\
\hline $\mathrm{SCAN}(\mathrm{PC})$ & $\underline{-0.13}$ & 4 & -0.10 & 5 & -0.09 & $\underline{11}$ & $\underline{-0.33}$ & 8 & $\underline{-0.26}$ & 10 \\
\hline $\mathrm{SCAN}(\mathrm{CR})$ & $\underline{-0.13}$ & 4 & -0.10 & 5 & $\underline{-0.12}$ & $\underline{12}$ & $\underline{-0.33}$ & 8 & $\underline{-0.26}$ & 10 \\
\hline SCAN(GEAloc) & -0.09 & 3 & -0.06 & 4 & 0.03 & 5 & 0.13 & -1 & 0.05 & 3 \\
\hline SCAN(PCopt) & $\underline{-0.12}$ & 3 & -0.08 & 4 & -0.04 & 8 & 0.07 & 0 & 0.01 & 4 \\
\hline SCAN(CRopt) & 0.03 & -1 & 0.05 & -2 & $\underline{0.16}$ & -5 & $\underline{0.41}$ & -9 & $\underline{0.36}$ & -5 \\
\hline TM & 6.63 & 29 & 6.49 & 32 & 5.73 & 44 & 14.17 & 50 & 13.21 & 35 \\
\hline TM(GEA2L) & -0.08 & 4 & -0.06 & 3 & -0.08 & 7 & -0.16 & 7 & -0.11 & 6 \\
\hline TM(TW02L) & -0.09 & 4 & -0.07 & 3 & -0.08 & 6 & -0.16 & 7 & -0.11 & 5 \\
\hline $\mathrm{TM}(\mathrm{PC})$ & $\underline{-0.15}$ & 4 & $\underline{-0.11}$ & 4 & -0.07 & 6 & -0.17 & 8 & -0.14 & 6 \\
\hline TM(CR) & -0.08 & 4 & -0.06 & 3 & -0.08 & 7 & -0.16 & 7 & -0.11 & 6 \\
\hline TM(GEAloc) & $\underline{-0.23}$ & $\underline{17}$ & $\underline{-0.21}$ & $\underline{15}$ & -0.07 & $\underline{15}$ & -0.14 & $\underline{14}$ & -0.15 & $\underline{13}$ \\
\hline TM(PCopt) & $\underline{-0.12}$ & 3 & -0.08 & 2 & 0.02 & 3 & 0.02 & 2 & 0.02 & 2 \\
\hline TM(CRopt) & -0.10 & 7 & -0.07 & 5 & 0.02 & 3 & 0.05 & 2 & 0.03 & 2 \\
\hline Reference & 6.70 & 48 & 6.69 & 40 & 5.71 & 95 & 13.97 & 111 & 12.96 & 93 \\
\hline
\end{tabular}

By choosing, again arbitrarily, $\sim 20 \%$ of the reference coupled cluster $[\mathrm{CCSD}(\mathrm{T})]$ or random-phase approximation (RPA) binding energy as the largest change that can be accepted when a functional is deorbitalized, then too large variations in $E_{\text {coh }}$ or $E_{\mathrm{b}}$ are usually observed for MVS, especially for the rare gases. The deorbitalization of SCAN or TM affects less the results, but nevertheless the change for the rare gases is in most cases also too large according to our criterion. Interestingly, note that the deorbitalization of the SCAN and TM functionals leads in many cases to a better agreement with $\operatorname{CCSD}(\mathrm{T})$ for the binding energy.

For the rare gases, the OF KED that leads overall to the smallest perturbations for the deorbitalization of the xc-MGGAs seems to be $t^{\mathrm{TW} 02 \mathrm{~L}}$. Note that $t^{\mathrm{CRopt}}$ shows rather strange results since it is the worst when used in MVS and SCAN, while it is the best for TM. In the case of the layered solids, a good choice for $t^{\mathrm{OF}}$ is $t^{\mathrm{GEAloc}}$ for MVS and SCAN, while with TM all $t^{\mathrm{OF}}$ except $t^{\mathrm{GEAloc}}$ are of similar accuracy.

\section{B. Band gaps}

Turning to the electronic structure, Table [V] and Fig. 2 (for selected methods) show the results obtained for the fundamental band gap $E_{\mathrm{g}}$ calculated with the mBJLDA potential and its deorbitalized versions. The testing set, which was used in our previous works $\underset{73,75}{\longrightarrow}$ consists of 76 solids (listed in Table S10 of the supplementary material ${ }^{83}$ ) of various types: ionic insulators, $s p$-semiconductors, rare gases, as well as strongly correlated solids. As shown in Refs. 73 and 75, the mBJLDA potential is on average more accurate for the band gap than all other semilocal potentials and hybrid functionals that were considered for comparison (the $\mathrm{PBE}^{9}$ and HSE06 ${ }^{98.99}$ results are also shown in Table IV and Fig.(2).

From the statistics shown in Table IV the first observa- 
TABLE IV. The ME, MAE, MRE, and MARE (with respect to experiment ${ }^{79,88}-97$ ) on the testing set of 76 solids (listed in Table S10 of the supplementary material ${ }^{83}$ ) for the fundamental band gap $E_{\mathrm{g}}$ obtained with mBJLDA and its deorbitalized versions, as well as PBE and HSE06. The units are eV for the ME and MAE and \% for the MRE and MARE.

\begin{tabular}{lcccc}
\hline \hline & ME & MAE & MRE & MARE \\
\hline mBJLDA & -0.30 & 0.47 & -5 & 15 \\
mBJLDA(GEA2L) & -0.95 & 0.97 & -32 & 32 \\
mBJLDA(TW02L) & -1.03 & 1.03 & -33 & 33 \\
mBJLDA(PC) & -1.17 & 1.18 & -32 & 33 \\
mBJLDA(CR) & -0.94 & 0.96 & -31 & 32 \\
mBJLDA(GEAloc) & 0.39 & 0.92 & 6 & 21 \\
mBJLDA(PCopt) & -0.54 & 0.67 & -10 & 16 \\
mBJLDA(CRopt) & -0.08 & 0.75 & -10 & 19 \\
PBE & -1.99 & 1.99 & -53 & 53 \\
HSE06 & -0.68 & 0.82 & -7 & 17 \\
\hline \hline
\end{tabular}

tion is that deorbitalizing the mBJLDA potential leads to an increase of the MAE and MARE, no matter what OF $\mathrm{KED}$ is used. The deterioration is the smallest when $t^{\mathrm{KS}}$ is replaced by $t^{\mathrm{PCopt}}$, and in this case the MAE increases from 0.47 to $0.67 \mathrm{eV}$ and the MARE from 15 to $16 \%$. This increase in the MARE is clearly negligible, but also quite acceptable for the MAE considering that most other potentials lead to larger MAE for this test set $\stackrel{73,75}{ }$ With mBJLDA(CRopt), a small increase of $4 \%$ for the MARE is obtained, while the MAE increases to $0.75 \mathrm{eV}$, which is now on the verge of being acceptable since other poten-

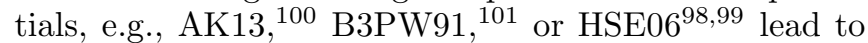
similar MAE $\underline{73}, 75$ Substituting $t^{\mathrm{KS}}$ by any of the other OF KED leads to a clearly larger MAE (around $1 \mathrm{eV}$ ) and MARE (above 30\%, except with $t^{\text {GEAloc }}$ ).

Looking into more detail at the results (see Table S11 and Figs. S25-S32 of the supplementary material 83 and Fig. 2), we can see that an inaccurate OF KED like $t^{\text {GEA2L }}$ leads to band gaps which are in most cases about halfway between the mBJLDA and PBE values, such that a rather clear underestimation is obtained on average (see ME and MRE in Table [V]). The mBJLDA band gaps are in general reproduced more accurately by $\mathrm{mB}-$ JLDA(PCopt) and/or mBJLDA(CRopt) except for the rare gases for which mBJLDA(GEA2L) is the closest to mBJLDA.

Finally, we note that a reoptimization of the parameters $\alpha$ and $\beta$ in a OF mBJLDA potential [see Ref. 32 for details] may possibly lead to a (partial) recovery of the performance of the original mBJLDA potential. However, we have not made any attempts since this is beyond the scope of this work.

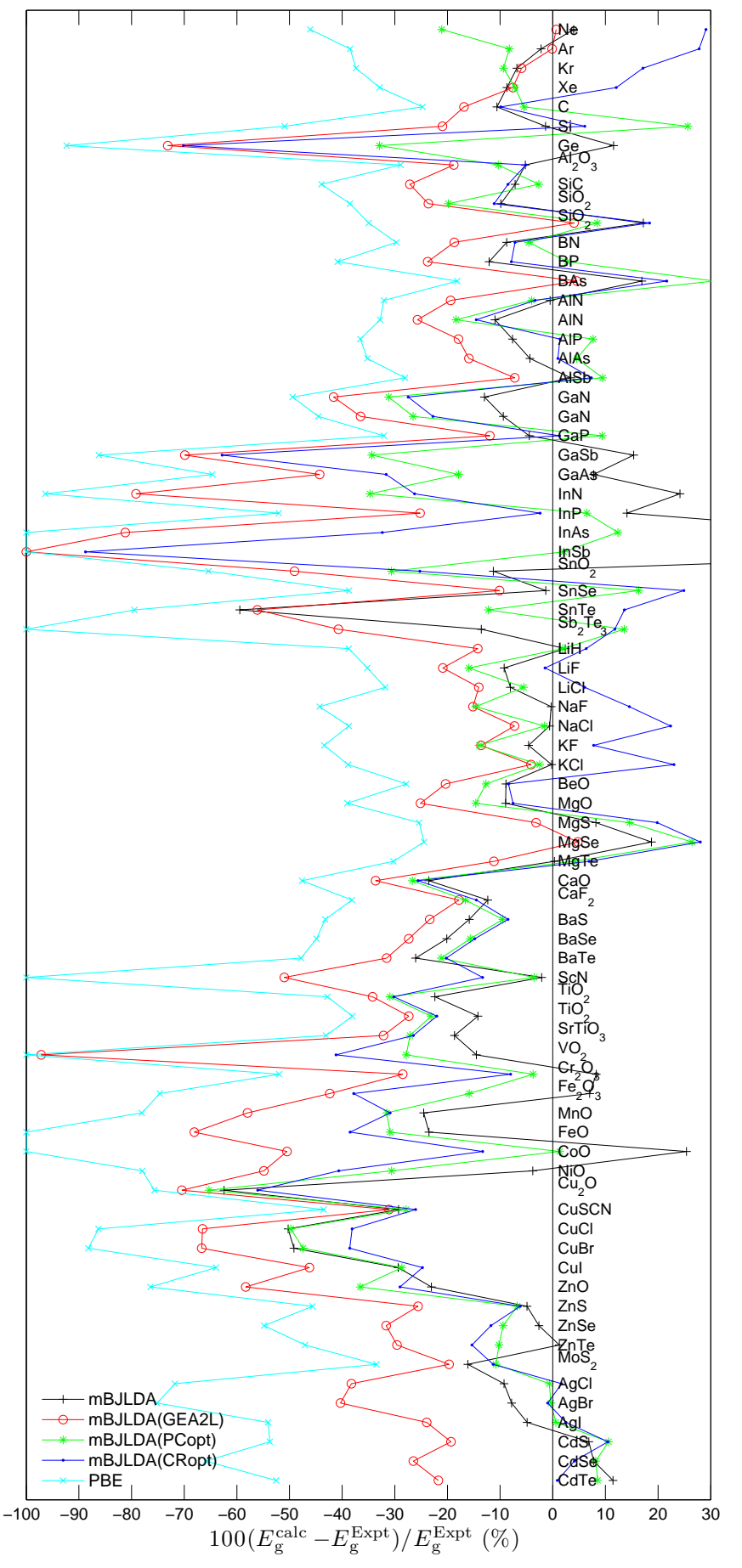

FIG. 2. Relative error (in \%) with respect to experiment ${ }^{79,88-97}$ in the band gap $E_{\mathrm{g}}$.

\section{FURTHER DISCUSSION}

Thanks to their additional dependency on $t^{\mathrm{KS}}, t^{\mathrm{KS}}$ MGGAs are more flexible than GGAs and, therefore, have the possibility to be universally more accurate. As shown above, a $t^{\mathrm{KS}}$-MGGA can be replaced rather efficiently (albeit not systematically) by a corresponding 


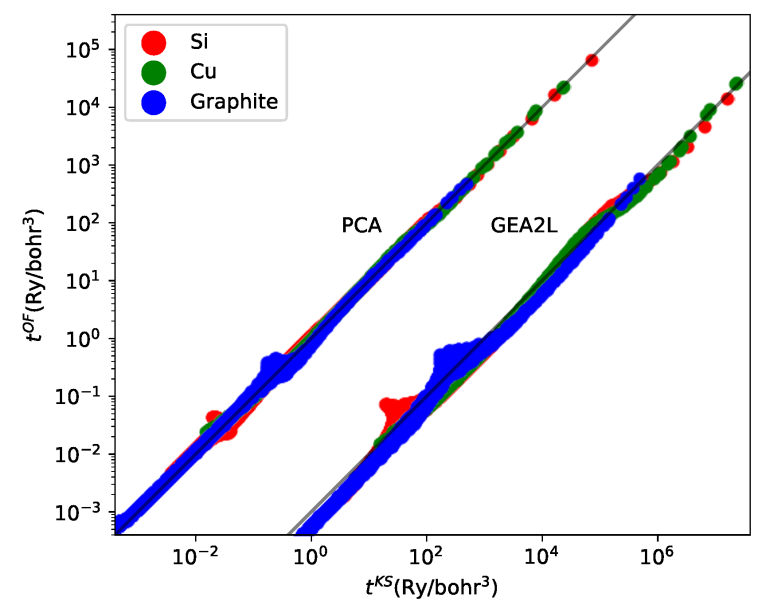

FIG. 3. Comparison between the KS KED and the GEA2L and PCA approximations for different solids. For clarity (no overlap between the GEA2L and PCA data), the $t^{\mathrm{KS}}$ values for GEA2L are multiplied by 1000 (i.e., right shifted). A perfect approximation should coincide with the diagonal solid black line.

$\nabla^{2} \rho$-MGGA, and in order to shed some light on the relation between $t^{\mathrm{KS}}$ and $\nabla^{2} \rho$, a principal component analysis $102.103(\mathrm{PCA})$ of $t^{\mathrm{TF}}, t^{\mathrm{W}}, \nabla^{2} \rho$, and $t^{\mathrm{KS}}$ has been carried out. From the PCA, an approximation for $t^{\mathrm{KS}}$ that consists of a linear combination of $t^{\mathrm{TF}}, t^{\mathrm{W}}$, and $\nabla^{2} \rho$ is obtained, and its accuracy reveals to which extent $t^{\mathrm{KS}}$ can be represented by $\rho$ and its first two derivatives.

The $4 \times 4$ covariance matrix was calculated using uniformly sampled data from one representative of metallic $(\mathrm{Cu})$, layered (graphite), and covalently bound $(\mathrm{Si})$ systems, and diagonalized in order to get the eigenvalues and corresponding eigenvectors spanning the fourdimensional space of $t^{\mathrm{TF}}, t^{\mathrm{W}}, \nabla^{2} \rho$, and $t^{\mathrm{KS}}$. In the next step, we neglect the eigenvector with the smallest eigenvalue, thereby obtaining the three dimensional representation which explains most of the variance in the data. Now, assuming that all points are on this three dimensional hyperplane, one can reconstruct an OF KED from the values of $\rho$ (i.e., $t^{\mathrm{TF}}$ ), $\nabla \rho$ (i.e., $t^{\mathrm{W}}$ ), and $\nabla^{2} \rho$, and the resulting linear combination is given by

$$
t^{\mathrm{PCA}}(\mathbf{r})=1.069 t^{\mathrm{TF}}(\mathbf{r})-0.244 t^{\mathrm{W}}(\mathbf{r})+0.438 \nabla^{2} \rho(\mathbf{r}) .
$$

The coefficient in front of $t^{\mathrm{TF}}$ is close to 1 as it should be in order to recover the homogeneous electron gas limit, while those in front of $t^{\mathrm{W}}$ and $\nabla^{2} \rho$ show big differences from GEA2L [Eq. (5)]. However, it is worth mentioning that a negative coefficient in front of $t^{\mathrm{W}}$ is found also in GEAloc $\frac{62}{2}(-0.165)$ and in a KED expression derived for the Airy gas $\frac{63}{}(-1 / 9 \approx-0.111)$.

Figure 3 shows for the three selected solids the accuracy of the GEA2L and our PCA approximation with the Weizsäcker lower bound enforced [Eq. [6)]. We can see that the PCA approximation shows better agreement with the KS KED than GEA2L, similarly as obtained (a)
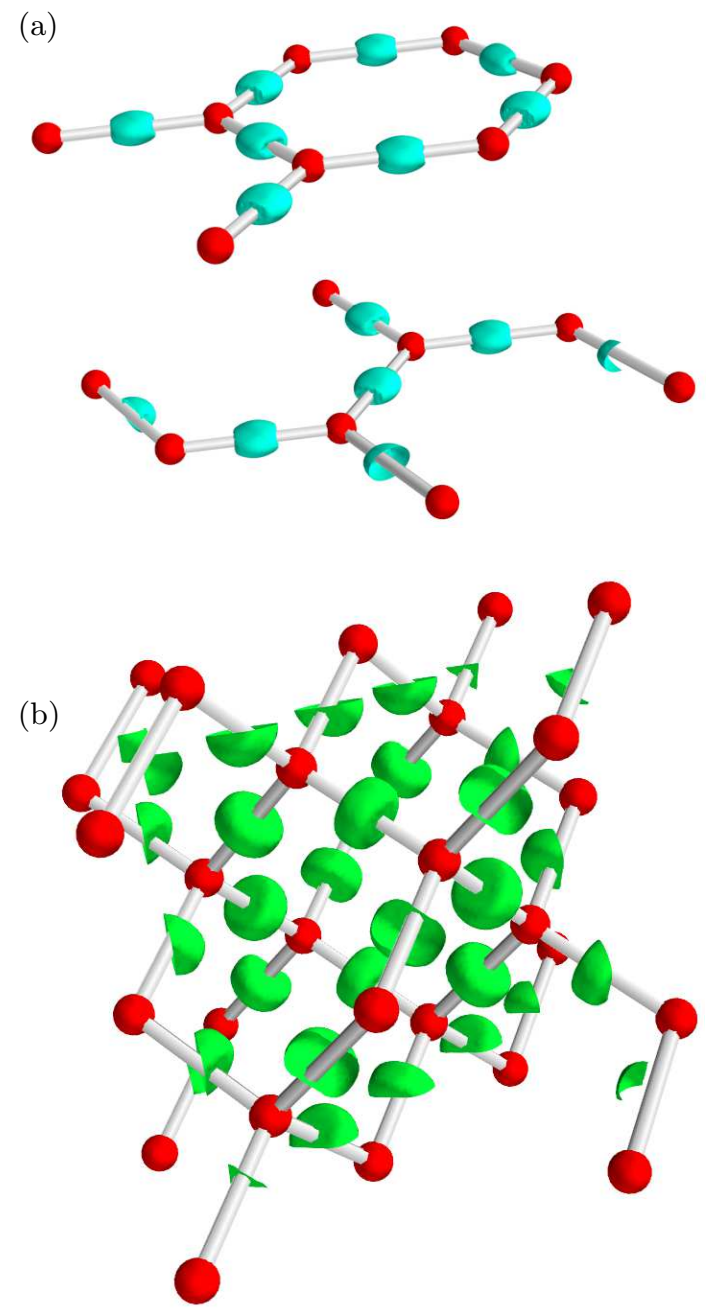

FIG. 4. Real space position of the lumps of Fig. 3. The atoms are represented by red spheres, while the erroneous points for (a) graphite (isosurface corresponding to $t^{\mathrm{GEA} 2 \mathrm{~L}} / t^{\mathrm{KS}}=2.25$ ) and (b) silicon (isosurface corresponding to $t^{\mathrm{GEA} 2 \mathrm{~L}} / t^{\mathrm{KS}}=1.9$ ) are in turquoise and green, respectively.

by Seino et al $\stackrel{46}{\underline{4}}$ for atoms and small organic molecules using a machine learning algorithm. It is also important to note that for both approximations there are two regions where one can find larger errors. These two lumps are from $\mathrm{Si}$ and graphite, where GEA2L systematically overestimates the KED, while in the PCA approximation these errors are still there but largely reduced. Actually, the errors for graphite can be found in the same KED region as the errors for organic molecules $\frac{46}{4}$

In Fig. 4, the erroneous points from these two regions are shown in real space, where we can see that the bigger errors occur in the middle of covalent bonds. If, for instance, for graphite the same PCA method is applied using only the points in the bonding regions, a much better accuracy (in these bonding regions) can be reached, 

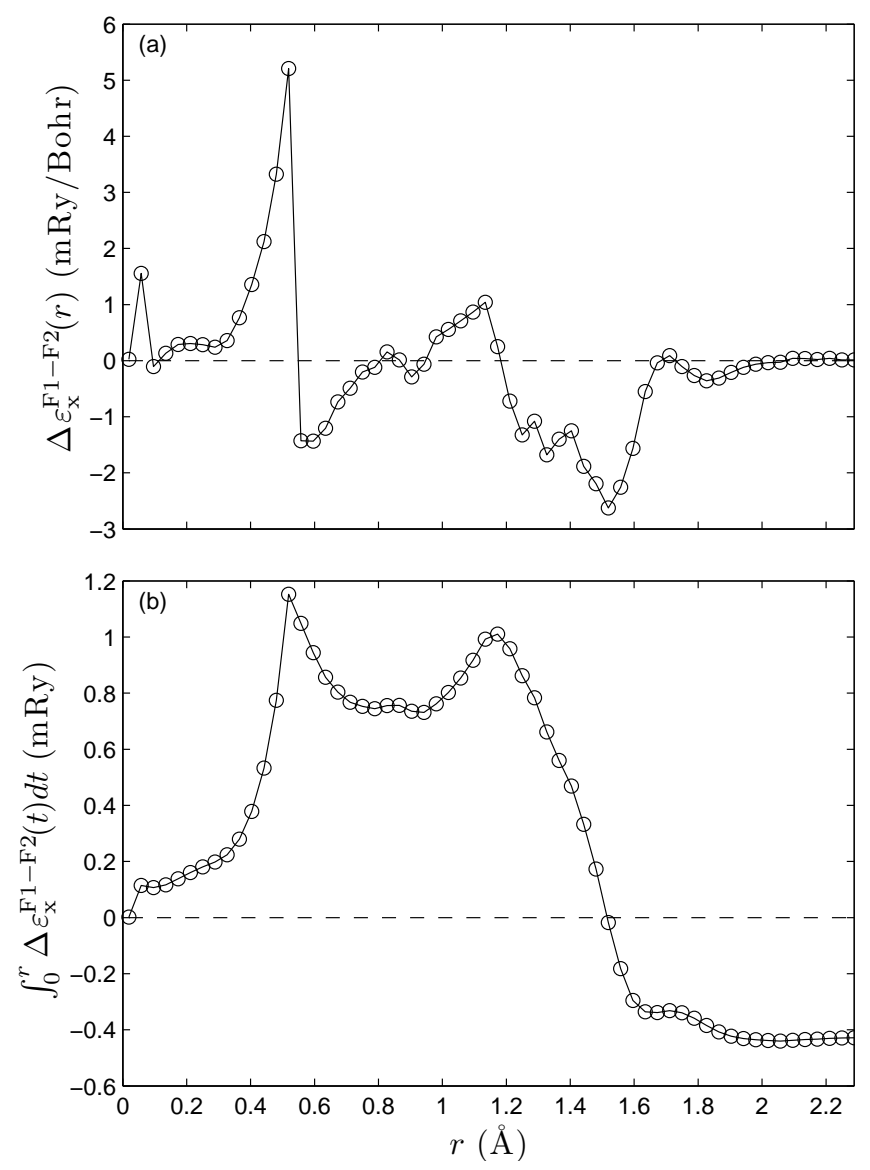

FIG. 5. Difference between the exchange components of SCAN(CRopt) (F1) and SCAN(GEA2L) (F2) in Si plotted as a function of the distance $r$ from an Si atom. Panel (a) shows the angular average of $\Delta \varepsilon_{\mathrm{x}}^{\mathrm{F} 1-\mathrm{F} 2}$ (see text for definition), while panel (b) shows the radial integration of $\Delta \varepsilon_{\mathrm{x}}^{\mathrm{F} 1-\mathrm{F} 2}$ from the atom until $r$.

and the resulting linear combination is given by

$$
t_{\text {bond }}^{\mathrm{PCA}}(\mathbf{r})=0.389 t^{\mathrm{TF}}(\mathbf{r})+0.635 t^{\mathrm{W}}(\mathbf{r})+0.084 \nabla^{2} \rho(\mathbf{r}) .
$$

While this is obviously not useful as a general KED approximation, it is interesting to note that $t^{\mathrm{W}}$ has now a small positive coefficient, in agreement with the fact that the covalent $\sigma$-bonding in graphite and silicon should be dominated by a single molecular orbital. As shown by Seino et al.,$\underline{46}$ considering also the third derivative of $\rho$ further improves the accuracy of OF KED. However, as discussed below, the bonding regions highlighted in Fig. 4 are not necessarily those which are the most relevant for explaining the differences observed in the results for the lattice constant.

In order to provide some insight into the results presented in Sec. III] Fig. 5 compares the energy density of SCAN(GEA2L) and SCAN(CRopt) in Si. For simplicity, only the exchange component, which is much larger than correlation, is considered. SCAN(GEA2L) and SCAN(CRopt) lead to rather different equilibrium lattice constants $a_{0}$ for $\mathrm{Si}$, namely, 5.437 and $5.460 \AA$,

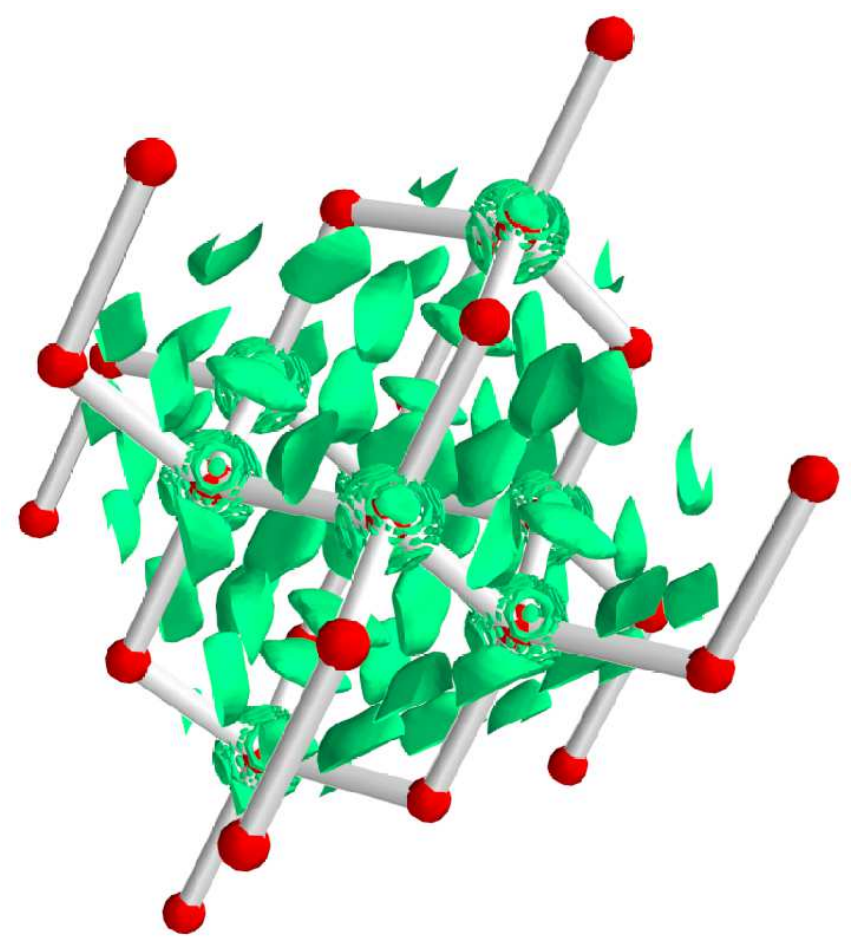

FIG. 6. Isosurface of the absolute value of $F_{\mathrm{x}}^{\mathrm{SCAN}(\mathrm{CRopt})}-$ $F_{\mathrm{x}}^{\mathrm{SCAN}(\mathrm{GEA} 2 \mathrm{~L})}$ corresponding to 0.03 .

respectively, and the following analysis provides details about the regions of space that are involved to explain these different values of $a_{0}$. Figure 5 (a) shows $\Delta \varepsilon_{\mathrm{x}}^{\mathrm{F} 1-\mathrm{F} 2}$, which is defined as

$$
\begin{aligned}
\Delta \varepsilon_{\mathrm{x}}^{\mathrm{F} 1-\mathrm{F} 2}(r)= & r^{2} \int\left[\left(\varepsilon_{\mathrm{x}}^{\mathrm{F} 1, a_{\text {large }}}(\mathbf{r})-\varepsilon_{\mathrm{x}}^{\mathrm{F} 1, a_{\text {small }}}(\mathbf{r})\right)\right. \\
& \left.-\left(\varepsilon_{\mathrm{x}}^{\mathrm{F} 2, a_{\text {large }}}(\mathbf{r})-\varepsilon_{\mathrm{x}}^{\mathrm{F} 2, a_{\text {small }}}(\mathbf{r})\right)\right] d \Omega,(9)
\end{aligned}
$$

where $\varepsilon_{\mathrm{x}}^{\mathrm{F}, a}$ is the exchange energy density [defined by Eq. (2)] of functional $\mathrm{F}$ (F1 and $\mathrm{F} 2$ designate SCAN(CRopt) and SCAN(GEA2L), respectively) calculated at a given lattice constant $\left(a_{\text {small }}\right.$ or $\left.a_{\text {large }}\right)$. The integration in Eq. (9) is over the spherical angles and $r$ is the distance from one $\mathrm{Si}$ atom. As discussed in detail in Refs. 104 and 105, the equilibrium lattice constant $a_{0}$ is determined by the slope of the xc-energy $E_{\mathrm{xc}}$, i.e., the variation of $E_{\mathrm{xc}}$ with respect to $a$, and this is basically what Fig. 5 shows since the difference between two values of $a$ ( $a_{\text {small }}$ and $\left.a_{\text {large }}\right)$ is considered. Figure 5 (b) shows the radial integration of $\Delta \varepsilon_{\mathrm{x}}^{\mathrm{F} 1 \mathrm{~F} 2}$ up to a given value of $r$. As already discussed in Ref. 104 for $\mathrm{Si}$ but in the case of GGA functionals, two different regions contribute significantly to the variation of $E_{\mathrm{xc}}$ with respect to $a$. The first one, located around $0.5 \AA$ [see the fast variations of the curves in Figs. [5(a) and (b)] corresponds to the core-valence separation. The second region extends from 1.2 to $1.7 \AA$ and corresponds to the valence/interstitial region which is rather large since $\mathrm{Si}$ has an open structure. Additionally, Fig. [6 


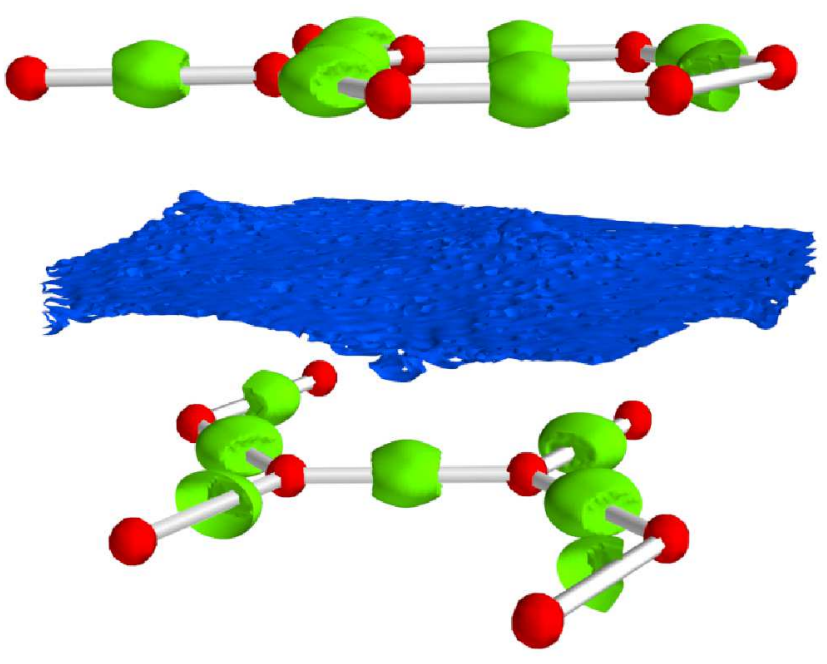

FIG. 7. The regions of space in graphite where $t^{\mathrm{GEA} 2 \mathrm{~L}} / t^{\mathrm{KS}}$ and $t^{\mathrm{KS}} / t^{\mathrm{GEA} 2 \mathrm{~L}}$ are larger than 1.9 are delimited by the isosurfaces in green and blue, respectively.

shows the isosurface of $\left|F_{\mathrm{x}}^{\mathrm{SCAN}(\mathrm{CRopt})}-F_{\mathrm{x}}^{\mathrm{SCAN}(\mathrm{GEA} 2 \mathrm{~L})}\right|$ that delimits values larger than 0.03 (where actually $\left.F_{\mathrm{x}}^{\mathrm{SCAN}(\mathrm{CRopt})}>F_{\mathrm{x}}^{\mathrm{SCAN}(\mathrm{GEA} 2 \mathrm{~L})}\right)$ and highlights the two types of regions just mentioned above.

The case of graphite was also discussed in Ref. 104, where the electron density and reduced density gradient $s$ in the region between the layers were studied in detail. It was shown that an increase of the interlayer distance leads to a rather large increase of $s$ overall, thus explaining the overestimation of the interlayer distance for GGA functionals with a too strong enhancement factor. Figure 7 shows the ratio $t^{\mathrm{GEA} 2 \mathrm{~L}} / t^{\mathrm{KS}}$ with a ratio that is smaller than the one used in Fig. 廿(a), such that the isosurface encloses a larger region. We can see that aside from the middle of the short covalent bonds within the layers (not relevant for the interlayer distance), also a non-negligible portion of the space between the layers has a ratio $\left(t^{\mathrm{KS}} / t^{\mathrm{GEA} 2 \mathrm{~L}}\right)$ bigger than 1.9.

In Sec. III, we also observed that an OF KED that is among the most accurate for a property calculated with the total energy, may be among the most inaccurate for the band gap, or vice versa. For instance, while $t^{\mathrm{PCopt}}$ and $t^{\mathrm{CRopt}}$ are not among the best KEDs for total-energy related properties of strongly bound solids, they are the most accurate for the band gap. Such contradictory results could seem quite puzzling at first sight, however this should be rather simple to explain in most cases.

Taking $\mathrm{LiH}$ as an example, Fig. 8 compares the xcenergy calculated with SCAN and selected deorbitalized SCANs by showing the difference $E_{\mathrm{xc}}^{t^{\mathrm{OF}}-\mathrm{SCAN}}-E_{\mathrm{xc}}^{t^{\mathrm{KS}}-\mathrm{SCAN}}$ as a function of the lattice constant $a$ (this is the same kind of analysis as the one used for Si in Fig. 51). Figures $8(a), 8(b)$, and $8(c)$ show the contributions from the $\mathrm{Li}$ atom, $\mathrm{H}$ atom, and interstitial region, respectively, while the sum of them (the total value in the unit cell)
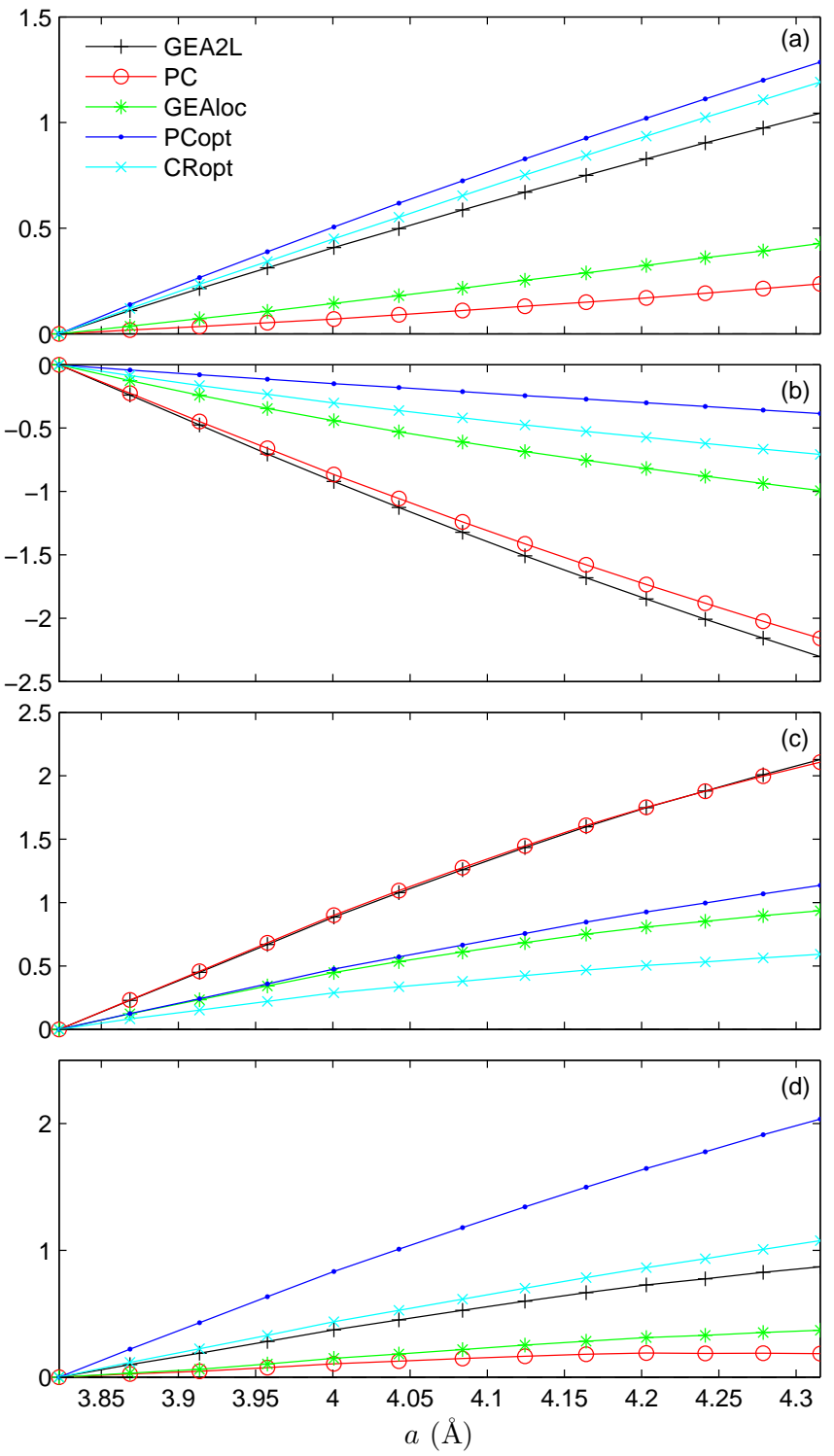

FIG. 8. Difference $E_{\mathrm{xc}}^{t^{\mathrm{OF}}-\mathrm{SCAN}}-E_{\mathrm{xc}}^{t^{\mathrm{KS}}{ }_{-\mathrm{SCAN}}}$ (in mRy) between the xc-energies of $\mathrm{LiH}$ obtained with SCAN and its deorbitalized versions plotted as a function of the lattice constant a. Panels (a), (b), and (c) show the contributions from the $\mathrm{Li}$ atom, $\mathrm{H}$ atom, and interstitial region, respectively, while panel (d) shows the sum of all contributions (i.e., the whole unit cell). The atomic muffin-tin spheres of the $\mathrm{Li}$ and $\mathrm{H}$ atoms are 1.7 Bohr. Each curve is vertically shifted such that the zero is at the smallest volume.

is shown in Fig. 8(d). As expected, the SCAN equilibrium lattice constants $a_{0}$ of $\mathrm{LiH}$ (see Table $\mathrm{S} 2$ of the supplementary material ${ }^{83}$ ) show the same ordering as the curves in Fig. 8(d) [the uppermost (lowest) curve correspond to the smallest (largest) lattice constant]. Thus, in the present case where the same functional is evaluated with different KED, the change in $a_{0}$ due to deorbitalization depends on the variation with $a$ of the difference between $t^{\mathrm{KS}}$ and $t^{\mathrm{OF}}$. From Fig. 8 , we can also see that 


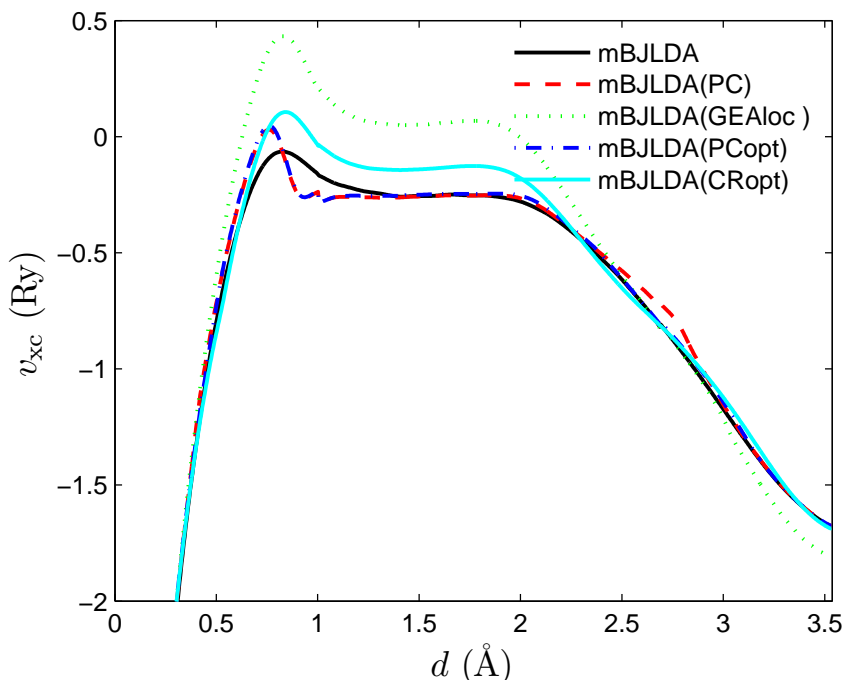

FIG. 9. mBJLDA xc-potential and a few selected of its deorbitalized versions plotted in $\mathrm{LiH}$ from the $\mathrm{Li}$ atom at $(0,0,0)$ to the $\mathrm{H}$ atom at $\left(\frac{1}{2}, \frac{1}{2}, \frac{1}{2}\right)$.

for all functionals, $E_{\mathrm{xc}}^{t^{\mathrm{OF}}-\mathrm{SCAN}}-E_{\mathrm{xc}}^{t^{\mathrm{KS}}-\mathrm{SCAN}}$ decreases in the $\mathrm{H}$ atom, but increases in the Li atom and interstitial region such that in total an increase is obtained. We also note that with $t^{\mathrm{GEA} 2 \mathrm{~L}}$ and $t^{\mathrm{PC}}$ there is a very large cancellation of the errors coming from the $\mathrm{H}$ atom and interstitial region.

As discussed in previous works, $\stackrel{70,106,107}{=}$ the magnitude of the band gap is determined by the inhomogeneities in the potential, such that, roughly speaking, large inhomogeneities favor larger values of the band gap. Actually, in most systems the valence band maximum and conduction band minimum are located close to an atom and in the interstitial region, respectively, which means that the difference in the magnitudes of a potential between these two regions determines the band gap. Again for LiH, Fig. 9 compares $v_{\mathrm{xc}}$ of mBJLDA and its $\mathrm{OF}$ variants. The $\mathrm{LiH}$ band gap (see Table S11 of the supplementary material ${ }^{83}$ ) with $\mathrm{mBJLDA}$ is $5.06 \mathrm{eV}$ and is reproduced at best by mBJLDA(PCopt) $(5.03 \mathrm{eV})$, while mBJLDA(GEAloc) with $6.69 \mathrm{eV}$ leads to the worst agreement. This is in accordance with Fig. 9, where we can see that the mBJLDA(PCopt) potential is the closest to $\mathrm{mB}$ JLDA, while the mBJLDA(GEAloc) potential is much higher in the interstitial region (where the conduction band minimum is located) and lower close to the $\mathrm{H}$ atom (where the valence band maximum is located).

Thus, from this detailed discussion about $\mathrm{LiH}$ it is rather clear that different mechanisms have to be invoked in order to explain the trends observed for the lattice constant (a total-energy related property) and band gap, such that opposite conclusions for these two types of properties can be obtained.

\section{SUMMARY}

In this work, the deorbitalization of several xc-MGGA methods, three energy functionals and one potential, has been investigated by considering properties of solids. The replacement $t^{\mathrm{KS}} \rightarrow t^{\mathrm{OF}}$ in xc-MGGAs affects the results to some degree which depends on both the xc-MGGA under investigation and the used approximation for the OF KED $t^{\mathrm{OF}}$.

Concerning the energy functionals for the calculation of the lattice constant, bulk modulus, and binding energy, we have shown that the results are in general more sensitive with MVS than with SCAN and TM, which should just be the direct consequence of the analytical form of the functionals that depends more strongly on the KED in the case of MVS. With SCAN and TM, the replacement $t^{\mathrm{KS}} \rightarrow t^{\mathrm{OF}}$ with most OF KED does not change much the results for strongly bound solids, such that the performance of a xc-MGGA remains pretty much the same. For the weakly bound rare gases, the change in the cohesive energy is usually rather large, while for the layered solids large changes in the interlayer distance are obtained with MVS.

The deorbitalization of the mBJLDA xc-potential leads to appreciable changes in the band gap and only the OF KED $t^{\mathrm{PCopt}}$ can be considered as a somehow reasonable replacement of $t^{\mathrm{KS}}$.

Similarly as Mejia-Rodriguez and Trickey ${ }^{22}$ we were not able to identify a OF KED that leads to reasonably small change in the results in most circumstances.

\section{ACKNOWLEDGMENTS}

This work was supported by the project F41 (SFB ViCoM) of the Austrian Science Fund (FWF) and by the TU-D doctoral college (TU Wien). F.T. acknowledges discussions with J. P. Perdew and L. A. Constantin.
1 P. Hohenberg and W. Kohn, Phys. Rev. 136, B864 (1964).

${ }^{2}$ W. Kohn and L. J. Sham, Phys. Rev. 140, A1133 (1965).

3 A. J. Cohen, P. Mori-Sánchez, and W. Yang, Chem. Rev. 112, 289 (2012).

4 A. D. Becke, J. Chem. Phys. 140, 18A301 (2014).

5 S. H. Vosko, L. Wilk, and M. Nusair, Can. J. Phys. 58, 1200 (1980).
6 J. P. Perdew and Y. Wang, Phys. Rev. B 45, 13244 (1992).

7 A. D. Becke, Phys. Rev. A 38, 3098 (1988).

8 C. Lee, W. Yang, and R. G. Parr, Phys. Rev. B 37, 785 (1988).

9 J. P. Perdew, K. Burke, and M. Ernzerhof, Phys. Rev. Lett. 77, 3865 (1996), 78, 1396(E) (1997).

10 J. P. Perdew, A. Ruzsinszky, G. I. Csonka, O. A. Vy- 
drov, G. E. Scuseria, L. A. Constantin, X. Zhou, and K. Burke, Phys. Rev. Lett. 100, 136406 (2008), 102, 039902(E) (2009); A. E. Mattsson, R. Armiento, and T. R. Mattsson, ibid. 101, 239701 (2008); J. P. Perdew, A. Ruzsinszky, G. I. Csonka, O. A. Vydrov, G. E. Scuseria, L. A. Constantin, X. Zhou, and K. Burke, ibid. 101, 239702 (2008).

11 F. Della Sala, E. Fabiano, and L. A. Constantin, Int. J. Quantum Chem. 116, 1641 (2016).

12 J. Sun, A. Ruzsinszky, and J. P. Perdew, Phys. Rev. Lett. 115, 036402 (2015).

13 J. Tao and Y. Mo, Phys. Rev. Lett. 117, 073001 (2016).

14 F. Tran, J. Stelzl, and P. Blaha, J. Chem. Phys. 144, 204120 (2016).

15 H. Peng, Z.-H. Yang, J. P. Perdew, and J. Sun, Phys. Rev. X 6, 041005 (2016).

16 Y. Mo, G. Tian, R. Car, V. N. Staroverov, G. E. Scuseria, and J. Tao, J. Chem. Phys. 145, 234306 (2016).

17 Y. Mo, R. Car, V. N. Staroverov, G. E. Scuseria, and J. Tao, Phys. Rev. B 95, 035118 (2017).

18 Y. Hinuma, H. Hayashi, Y. Kumagai, I. Tanaka, and F. Oba, Phys. Rev. B 96, 094102 (2017).

19 P. J̈emmer and P. J. Knowles, Phys. Rev. A 51, 3571 (1995).

20 R. Neumann and N. C. Handy, Chem. Phys. Lett. 266, 16 (1997).

21 A. C. Cancio, C. E. Wagner, and S. A. Wood, Int. J. Quantum Chem. 112, 3796 (2012).

22 D. Mejia-Rodriguez and S. B. Trickey, Phys. Rev. A 96, 052512 (2017).

23 S. Laricchia, L. A. Constantin, E. Fabiano, and F. Della Sala, J. Chem. Theory Comput. 10, 164 (2014).

${ }^{24}$ R. Neumann, R. H. Nobes, and N. C. Handy, Mol. Phys. 87, 1 (1996).

25 J. A. Pople, P. M. W. Gill, and B. G. Johnson, Chem. Phys. Lett. 199, 557 (1992).

26 L. Ferrighi, G. K. H. Madsen, and B. Hammer, J. Chem. Phys. 135, 084704 (2011).

27 J. Sun, M. Marsman, G. I. Csonka, A. Ruzsinszky, P. Hao, Y.-S. Kim, G. Kresse, and J. P. Perdew, Phys. Rev. B 84, 035117 (2011).

28 J. C. Womack, N. Mardirossian, M. Head-Gordon, and C.-K. Skylaris, J. Chem. Phys. 145, 204114 (2016).

29 Y. Yao and Y. Kanai, J. Chem. Phys. 146, 224105 (2017).

30 A. D. Becke and K. E. Edgecombe, J. Chem. Phys. 92, 5397 (1990).

31 A. V. Bienvenu and G. Knizia, J. Chem. Theory Comput. 14, 1297 (2018).

32 F. Tran and P. Blaha, Phys. Rev. Lett. 102, 226401 (2009).

33 V. L. Lignères and E. A. Carter, Handbook of Materials Modeling, edited by S. Yip (Springer, Dordrecht, 2005) p. 137.

34 T. A. Wesolowski and Y. A. Wang, eds., Recent Progress in Orbital-free Density Functional Theory (World Scientific, Singapore, 2013).

35 V. V. Karasiev and S. B. Trickey, Adv. Quantum Chem. 71, 221 (2015).

36 C. R. Jacob and J. Neugebauer, WIREs Comput. Mol. Sci. 4, 325 (2014).

37 T. A. Wesolowski, S. Shedge, and X. Zhou, Chem. Rev. 115, 5891 (2015).

38 S. Śmiga, E. Fabiano, L. A. Constantin, and
F. Della Sala, J. Chem. Phys. 146, 064105 (2017).

39 K. Jiang, J. Nafziger, and A. Wasserman, J. Chem. Phys. 148, 104113 (2018).

40 L. H. Thomas, Proc. Cambridge Philos. Soc. 23, 542 (1927).

41 E. Fermi, Rend. Accad. Naz. Lincei 6, 602 (1927).

42 R. G. Parr and W. Yang, Density-Functional Theory of Atoms and Molecules (Oxford University Press, New York, 1989).

43 A. J. Thakkar, Phys. Rev. A 46, 6920 (1992).

44 S. S. Iyengar, M. Ernzerhof, S. N. Maximoff, and G. E. Scuseria, Phys. Rev. A 63, 052508 (2001).

45 F. Tran and T. A. Wesołowski, Chem. Phys. Lett. 360, 209 (2002).

46 J. Seino, R. Kageyama, M. Fujinami, Y. Ikabata, and H. Nakai, J. Chem. Phys. 148, 241705 (2018).

47 F. Tran and T. A. Wesołowski, Int. J. Quantum Chem. 89, 441 (2002).

48 D. García-Aldea and J. E. Alvarellos, J. Chem. Phys. 127, 144109 (2007).

${ }^{49}$ V. V. Karasiev, S. B. Trickey, and F. E. Harris, J. Comput.-Aided Mater. Des. 13, 111 (2006).

50 F. Tran and T. A. Wesolowski, Recent Progress in Orbitalfree Density Functional Theory (World Scientific, Singapore, 2013) p. 429.

51 N. H. March and R. Santamaria, Int. J. Quantum Chem. 39, 585 (1991).

52 H. Lee, C. Lee, and R. G. Parr, Phys. Rev. A 44, 768 (1991).

53 J. A. Alonso and L. A. Girifalco, Chem. Phys. Lett. 53, 190 (1978).

54 W. Yang, R. G. Parr, and C. Lee, Phys. Rev. A 34, 4586 (1986).

55 K. Finzel, Theor. Chem. Acc. 134, 106 (2015).

56 A. C. Cancio, D. Stewart, and A. Kuna, J. Chem. Phys. 144, 084107 (2016).

57 D. A. Kirzhnits, Sov. Phys. JETP 5, 64 (1957).

58 M. Brack, B. K. Jennings, and Y. H. Chu, Phys. Lett. B 65, 1 (1976).

59 C. F. von Weizsäcker, Z. Phys. 96, 431 (1935).

60 J. P. Perdew and L. A. Constantin, Phys. Rev. B 75, 155109 (2007).

61 E. X. Salazar, P. F. Guarderas, E. V. Ludeña, M. H. Cornejo, and V. V. Karasiev, Int. J. Quantum Chem. 116, 1313 (2016).

62 A. C. Cancio and J. J. Redd, Mol. Phys. 115, 618 (2017).

63 A. Lindmaa, A. E. Mattsson, and R. Armiento, Phys. Rev. B 90, 075139 (2014), 95, 079902(E) (2017).

64 A. A. Astakhov, A. I. Stash, and V. G. Tsirelson, Int. J. Quantum Chem. 116, 237 (2016).

65 M. Hoffmann-Ostenhof and T. Hoffmann-Ostenhof, Phys. Rev. A 16, 1782 (1977).

66 S. Kurth, J. P. Perdew, and P. Blaha, Int. J. Quantum Chem. 75, 889 (1999).

67 G. L. Oliver and J. P. Perdew, Phys. Rev. A 20, 397 (1979).

68 J. Sun, J. P. Perdew, and A. Ruzsinszky, Proc. Natl. Acad. Sci. U.S.A. 112, 685 (2015).

69 A. D. Becke and E. R. Johnson, J. Chem. Phys. 124, 221101 (2006).

70 F. Tran, P. Blaha, and K. Schwarz, J. Phys.: Condens. Matter 19, 196208 (2007).

71 D. J. Singh, Phys. Rev. B 82, 205102 (2010).

72 H. Jiang, J. Chem. Phys. 138, 134115 (2013). 
73 F. Tran and P. Blaha, J. Phys. Chem. A 121, 3318 (2017).

${ }^{74}$ K. Nakano and T. Sakai, J. Appl. Phys. 123, 015104 (2018).

75 F. Tran, S. Ehsan, and P. Blaha, Phys. Rev. Materials 2, 023802 (2018).

76 P. Blaha, K. Schwarz, G. K. H. Madsen, D. Kvasnicka, J. Luitz, R. Laskowski, F. Tran, and L. D. Marks, WIEN2K: An Augmented Plane Wave plus Local Orbitals Program for Calculating Crystal Properties (Vienna University of Technology, Austria, 2018).

77 O. K. Andersen, Phys. Rev. B 12, 3060 (1975).

78 D. J. Singh and L. Nordström, Planewaves, Pseudopotentials, and the LAPW Method, 2nd ed. (Springer, New York, 2006).

${ }^{79}$ L. Schimka, J. Harl, and G. Kresse, J. Chem. Phys. 134, 024116 (2011).

80 K. Lejaeghere, V. Van Speybroeck, G. Van Oost, and S. Cottenier, Crit. Rev. Solid State Mater. Sci. 39, 1 (2014).

81 K. Rościszewski, B. Paulus, P. Fulde, and H. Stoll, Phys. Rev. B 62, 5482 (2000).

82 T. Björkman, Phys. Rev. B 86, 165109 (2012).

83 See supplementary material at http://dx.doi.org/ for the detailed results for the lattice constant, bulk modulus, cohesive energy, and band gap.

84 E. R. Johnson, R. A. Wolkow, and G. A. DiLabio, Chem. Phys. Lett. 394, 334 (2004).

${ }^{85}$ Y. Zhao and D. G. Truhlar, J. Phys. Chem. A 110, 5121 (2006).

86 F. Tran and J. Hutter, J. Chem. Phys. 138, 204103 (2013), 139, 039903 (2013).

87 C. R. C. Rêgo, L. N. Oliveira, P. Tereshchuk, and J. L. F. Da Silva, J. Phys.: Condens. Matter 27, 415502 (2015), 28, 129501 (2016).

88 J. M. Crowley, J. Tahir-Kheli, and W. A. Goddard, III, J. Phys. Chem. Lett. 7, 1198 (2016).
${ }^{89}$ M. J. Lucero, T. M. Henderson, and G. E. Scuseria, J. Phys.: Condens. Matter 24, 145504 (2012).

90 S. Bernstorff and V. Saile, Opt. Commun. 58, 181 (1986).

91 R. Gillen and J. Robertson, J. Phys.: Condens. Matter 25, 165502 (2013).

92 D. Koller, P. Blaha, and F. Tran, J. Phys.: Condens. Matter 25, 435503 (2013).

93 J. H. Skone, M. Govoni, and G. Galli, Phys. Rev. B 89, 195112 (2014).

94 H. Shi, R. I. Eglitis, and G. Borstel, Phys. Rev. B 72, 045109 (2005).

95 J. Lee, A. Seko, K. Shitara, K. Nakayama, and I. Tanaka, Phys. Rev. B 93, 115104 (2016).

96 A. M. Ganose and D. O. Scanlon, J. Mater. Chem. C 4, 1467 (2016)

97 D. Groh, R. Pandey, M. B. Sahariah, E. Amzallag, I. Baraille, and M. Rérat, J. Phys. Chem. Solids 70, 789 (2009).

98 J. Heyd, G. E. Scuseria, and M. Ernzerhof, J. Chem. Phys. 118, 8207 (2003), 124, 219906 (2006).

99 A. V. Krukau, O. A. Vydrov, A. F. Izmaylov, and G. E. Scuseria, J. Chem. Phys. 125, 224106 (2006).

100 R. Armiento and S. Kümmel, Phys. Rev. Lett. 111, 036402 (2013).

101 A. D. Becke, J. Chem. Phys. 98, 5648 (1993).

102 K. Pearson, Lond. Edinb. Dublin Philos. Mag. J. Sci. 2, 559 (1901).

103 I. T. Jolliffe, Principal Component Analysis, 2nd ed. (Springer, New York, 2002).

104 P. Haas, F. Tran, P. Blaha, K. Schwarz, and R. Laskowski, Phys. Rev. B 80, 195109 (2009).

105 H. Levämäki, M. P. J. Punkkinen, K. Kokko, and L. Vitos, Phys. Rev. B 89, 115107 (2014).

106 M. Städele, J. A. Majewski, P. Vogl, and A. Görling, Phys. Rev. Lett. 79, 2089 (1997).

107 F. Tran, P. Blaha, and K. Schwarz, J. Chem. Theory Comput. 11, 4717 (2015). 